\title{
Population genomics provides insights in diversity, epidemiology, evolution and pathogenicity of the waterborne pathogen Mycobacterium kansasii
}

Tao Luo

Sichuan University

Peng Xu

Zunyi Medical University

Yangyi Zhang

Shanghai Municipal Centre for Disease Control and Prevention

Jessica L. Porter

University of Melbourne

Marwan Ghanem

McGill University and McGill International TB Centre

Qingyun Liu

Fudan University https://orcid.org/0000-0002-2284-5050

Yuan Jiang

Shanghai Municipal Centre for Disease Control and Prevention

Jing Li

Shanghai Municipal Centre for Disease Control and Prevention

Qing Miao

Fudan University

Bijie Hu

Fudan University

Benjamin P. Howden

University of Melbourne https://orcid.org/0000-0003-0237-1473

Janet Fyfe

Victorian Infectious Diseases Reference Laboratory

Maria Globan

Melbourne Health

Wencong $\mathrm{He}$

Chinese Center for Disease Control and Prevention

\section{Ping $\mathrm{He}$}

Chinese Center for Disease Control and Prevention

\section{Yiting Wang}


Chinese Center for Disease Control and Prevention

\section{Houming Liu}

Chinese Center for Disease Control and Prevention

\section{Howard Takiff}

Institut Pasteur

\section{Yanlin Zhao}

Chinese Center for Disease Control and Prevention https://orcid.org/0000-0002-7890-4312

\section{Xinchun Chen}

Shenzhen University

\section{Qichao Pan}

Shanghai Municipal Centre for Disease Control and Prevention

\section{MA Behr}

McGill University

\section{Timothy Stinear}

University of Melbourne https://orcid.org/0000-0003-0150-123X

\section{Qian Gao ( $\nabla$ qiangao@fudan.edu.cn )}

Fudan University

\section{Article}

Keywords: Mycobacterium kansasii, population genomics, epidemiology

Posted Date: October 1st, 2020

DOI: https://doi.org/10.21203/rs.3.rs-78954/v1

License: (c) (i) This work is licensed under a Creative Commons Attribution 4.0 International License. Read Full License

Version of Record: A version of this preprint was published at Nature Communications on May 3rd, 2021. See the published version at https://doi.org/10.1038/s41467-021-22760-6. 
1 Population genomics provides insights in diversity, epidemiology, evolution and

4 \#Tao Luo ${ }^{1,2}$, \#Peng Xu ${ }^{2,3}$, Yangyi Zhang ${ }^{4}$, Jessica L. Porter ${ }^{5,}$, Marwan Ghanem ${ }^{7}$, Qingyun

5 Liu $^{2}$, Yuan Jiang ${ }^{4}$, Jing $\mathrm{Li}^{4}$, Qing Miao ${ }^{8}$, Bijie Hu ${ }^{8}$, Benjamin P. Howden ${ }^{5,6,9}$, Janet A. M.

6 Fyfe ${ }^{10}$, Maria Globan ${ }^{10}$, Wencong $\mathrm{He}^{11}$, Ping $\mathrm{He}^{11}$, Yiting Wang ${ }^{11}$, Houming Liu ${ }^{12}$, Howard E.

7 Takiff ${ }^{13}$, *Yanlin Zhao ${ }^{11}$, *Xinchun Chen ${ }^{14}$, *Qichao Pan ${ }^{4},{ }^{*}$ Marcel A. Behr ${ }^{7},{ }^{*}$ Tim P. Stinear

$8 \quad 5,6, *$ Qian $\mathrm{Gao}^{2}$

10 1. Department of Pathogenic Biology, West China School of Basic Medical Sciences \& Forensic

11 Medicine, Sichuan University, Chengdu, China.

12 2. Key Laboratory of Medical Molecular Virology (MOE/NHC/CAMS), Shanghai Medical

13 College and School of Basic Medical Sciences, Shanghai Public Health Clinical Center, Fudan

14 University, Shanghai, China.

15 3. Key Laboratory of Characteristic Infectious Disease \& Bio-safety Development of Guizhou

16 Province Education Department, Institute of Life Sciences, Zunyi Medical University, Zunyi,

17 China

18 4. Department of Tuberculosis Control, Shanghai Municipal Centre for Disease Control and

19 Prevention, Shanghai, China

20 5. Department of Microbiology and Immunology, Doherty Institute for Infection and Immunity,

21 University of Melbourne, Melbourne, Victoria 3000, Australia

22 6. Doherty Applied Microbial Genomics, Doherty Institute for Infection and Immunity,

23 University of Melbourne, Melbourne, Victoria 3000, Australia

24 7. Department of Microbiology and Immunology, McGill University and McGill International

25 TB Centre, Montreal, Quebec, Canada

26 8. Department of Infectious Diseases, Zhongshan Hospital, Fudan University, Shanghai, China 
27 9. Microbiological Diagnostic Unit Public Health Laboratory, Doherty Institute for Infection and

28 Immunity, University of Melbourne, Melbourne, Victoria 3000, Australia

29 10. Victorian Infectious Diseases Reference Laboratory, Doherty Institute for Infection and

30 Immunity, Melbourne Health, Melbourne, Victoria 3000, Australia

31 11. Chinese Center for Disease Control and Prevention, and Beijing Tuberculosis and Thoracic

32 Tumor Research Institute, Beijing, China

33 12. Department of Clinical Laboratory, The Third People's Hospital of Shenzhen, Southern

34 University of Science and Technology, Shenzhen, China

35 13. Integrated Mycobacterial Pathogenomics Unit, Institut Pasteur, Paris, France.

36 14. Guangdong Provincial Key Laboratory of Regional Immunity and Diseases, Department of

37 Pathogen Biology, Shenzhen University School of Medicine, Shenzhen 518055, China

$39{ }^{*}$ Contribute equally

$40 *$ Correspondence to:

41 Qian Gao (qiangao@,fudan.edu.cn)

42 Tim P. Stinear (tstinear@unimelb.edu.au)

43 Marcel A. Behr (marcel.behr@mcgill.ca)

44 Qichao Pan (qcpan@scdc.sh.cn)

45 Xinchun Chen (chenxinchun@szu.edu.cn)

46 Yanlin Zhao (zhaoyl@,chinacdc.cn) 


\section{Abstract}

48 Mycobacterium kansasii is a nontuberculous mycobacterium that can cause serious pulmonary

49 disease. Genotyping suggested that the species is composed of at least six subtypes that vary in

50 clinical significance, with subtype I being clinically dominant but less commonly isolated from

51 environmental sources. Here we report a population genomics study of 358 M. kansasii isolates

52 obtained from global water and clinical sources. Phylogenomic analyses revealed that the six

53 subtypes are more accurately designated as closely related subspecies. These subspecies show

54 ample evidence of recombination mediated by distributive conjugative transfer that has

55 contributed to subspeciation and on-going diversification. Water was confirmed as a source of

56 clinical infections by showing that genomes of clinical strains from an Australian outbreak were

57 almost indistinguishable from strains contaminating the drinking water supply. Most clinical

58 infections (nearly $80 \%$ ) were due to a recently emerged group of strains designated the $M$.

59 kansasii main complex (MKMC), which appears to have originated in Europe in 1900s and

60 expanded globally over the past century. Comparative genomic analyses revealed that the

61 MKMC has maintained the methylcitrate cycle and expanded ESX-I secretion-associated

62 proteins, perhaps facilitating metabolic adaptation and pathogenicity for human hosts. Evidence

63 of on-going positive selection in isolates of the MKMC was found in genes involved in carbon

64 and secondary metabolism, metal ion homeostasis and cell surface remodeling that could

65 represent adaptation to human hosts. These results further our understanding of the epidemiology

66 and pathogenicity of M. kansasii and emphasize the importance of monitoring its potential

67 transition to a more human-adapted pathogen. 
Nontuberculous mycobacteria (NTM) are environmental bacteria and some species can cause opportunistic infections in humans. While they are not as pathogenic as M. tuberculosis,

71 diseases due to NTM have been an increasing concern in global health ${ }^{1-4}$, and in some developed

72 countries NTM are now responsible for more disease than M. tuberculosis ${ }^{2,4}$. M. kansasii is

73 among the most pathogenic NTM and has the highest clinical relevance ${ }^{5}$. It is one of the last

74 species to have diverged from a common ancestor before the appearance of the M. tuberculosis

75 complex ${ }^{6}$, and is capable of causing aggressive and destructive pulmonary disease resembling

76 tuberculosis ${ }^{7}$. In the mid-20 $0^{\text {th }}$ century, before the emergence of the HIV pandemic, M. kansasii

77 was dominant among NTM diseases in several regions of United States, Europe and Japan ${ }^{3}$. It is

78 currently one of the most frequent causes of NTM pulmonary disease throughout the world (Fig.

79 1A), with a relatively high incidence in regions of Europe, South America, Africa and Asia ${ }^{3,8}$. In

80 China, M. kansasii has been isolated from pulmonary infections in many areas, but the incidence

81 is highest in the highly urbanized eastern and southern coastal regions ${ }^{9-12}$. From 2008 to 2012 in

82 Shanghai, it was responsible for nearly half of all NTM infections ${ }^{13}$.

As with other NTM, M. kansasii infections are generally assumed to be acquired from

84 environmental sources rather than by human-to-human transmission. Although municipal water

85 distribution systems are believed to be the major reservoir for human M. kansasii infections ${ }^{3,5,14}$,

86 water isolates are usually genetically distinct from clinical strains. Molecular typing has revealed

87 that M. kansasii comprises at least six distinct subtypes that vary in prevalence and clinical

88 relevance ${ }^{15-18}$. M. kansasii subtype I is responsible for the vast majority of infections worldwide

89 but is not often isolated from water sources ${ }^{15,17,18}$. Until now, no definitive epidemiological link

90 has been established between water reservoirs and clinical infections ${ }^{19,20}$. Instead, genotyping

91 has shown that clinical strains of M. kansasii subtype I isolated from diverse geographic locations

92 constitute a homogenous population ${ }^{15,18}$, suggesting potential human-to-human transmission of a

93 successfully clone. Actually, potential transmission of M. kansasii between family members has 
94 been reported in several cases ${ }^{21,22}$. In addition, transmission has been recently revealed as a

95 major route for the dissemination of dominant clones of another NTM, the M. abscessus ${ }^{23}$.

96 Consistent with the clinical dominance, M. kansasii subtype I showed the highest clinical

97 relevance, as it has been associated with severe and even fatal disease in both immune-competent

98 and immune-compromised patients, while the other subtypes are isolated only from immune-

99 compromised patients or environmental sources ${ }^{17}$. Although subtype I causes more disease than

100 the other subtypes, the genetic determinants of its pathogenic adaptation have not been addressed.

101 In addition, clinical M. kansasii isolates can vary phenotypically, with strains showing either a

102 smooth or rough colony morphology due to difference in cell wall hydrophobicity ${ }^{24,25}$. Similar to

103 M. abscessus ${ }^{26}$, M. kansasii strains with the rough colony appear to be more virulent which could

104 establish chronic systemic infections in mouse ${ }^{25,27}$, but the genetic basis for the phenotypic

105 differences has not been explained.

106 In the current study, we analyzed the genomes of a worldwide collection of isolates to

107 better define the global population structure of M. kansasii. The genomic analyses provided

108 insights into its speciation, diversification, the sources of clinical infections and possible genetic

109 determinants associated with its ability to proliferation and cause disease in human.

\section{Results}

\section{Global diversity of $M$. kansasii}

113 We performed whole genome sequencing on 271 M. kansasii isolates, including 155

114 isolates from China, 74 isolates from Australia, 34 clinical isolates from European and North

115 American countries, 5 from South Africa and 3 from Japan. These genomes, together with an

116 additional 84 M. kansasii genomes available from public databases (Supplementary Table 1),

117 were analyzed for global diversity. Given the close genetic relationship with M. kansasii, three

118 M. gastri genomes (one sequenced in the current study) were also included. In total, we included

119 the genomes of 358 isolates obtained from 18 countries with varying burdens of disease caused 
120 by $M$. kansasii (Fig. 1A). On average, the $M$. kansasii genomes are $6.29 \mathrm{Mb}$ in length and contain

1215,757 protein coding genes. A genomic alignment of 2,280 core genes with at least $90 \%$ amino

122 acid identity between strains and covering $2.12 \mathrm{M}$ nucleotides was used to generate a maximum

123 likelihood phylogeny (Fig. 1B). The phylogeny consisted of seven distinct lineages

124 corresponding to the six M. kansasii subtypes and M. gastri. The pairwise genome-wide average

125 nucleotide identities (gANI) within each lineage were all over 98\%, while the gANIs between

126 lineages were all below 95\% (Fig. 1C), suggesting that the distinct lineages should be regarded as

127 different subspecies rather than subtypes ${ }^{28,29}$. The $M$. gastri strains were closely related to $M$.

128 kansasii ssp. IV, and were therefore designated as a seventh subspecies of M. kansasii. Four

129 subspecies (I, II, III and VI), each containing more than ten strains, were designated as the major

130 subspecies in the current study. Pairwise comparison of single nucleotide variants (SNV)

131 amongst the strains of each of the four major subspecies revealed a median difference of 129 to

1329,137 SNVs along the 2.12Mbp core genome (Fig. S1). To determine the general evolutionary

133 forces that shaped the diversity of M. kansasii subspecies, we calculated Tajima's D statistics for

134 the major subspecies along the core genome (Fig. S2). The median values for ssp. II, III and VI

$135(0.17,0.72$ and 0.16 respectively) indicated balancing selection, while the median value for the

136 dominant ssp. I (-2.68) suggested recent population expansion and a potential selective sweep.

\section{Recombination driving subspeciation and diversification of $M$. kansasii subspecies}

Alignment of the $16 \mathrm{~S}, 23 \mathrm{~S}$ rRNA and spacer region revealed several sequence mosaics

140 that infer a reticulate network for the M. kansasii subspecies (Fig. S3), consistent with

141 evolutionary processes in the presence of recombination. A more complex network was obtained

142 based on 378,876 SNVs along the core genome alignment (Fig. 2A), suggesting that

143 recombination has occurred across the whole genome. Analysis of the core-genome alignment

144 with the fastGEAR algorithm identified seven population clusters corresponding to the seven

145 subspecies, with extensive ancestral recombination (occurring during the subspeciation) and 
146 recent recombination (occurring after the subspeciation) between subspecies that resulted in

147 highly mosaic genomes (Fig. 2B and Fig. S4). An average of $411 \mathrm{~Kb}(18.4 \%)$ of the core

148 genome was involved in ancestral recombination that contributed to the origin of the subspecies.

149 Recent recombination was detected in all subspecies, with the total genomic fraction of

150 recombinant fragments varying from 0 to $12.2 \%$ amongst strains of the different subspecies. For

151 the recent recombination events, most recombinant fragments share high identity ( $\geq 99 \%)$ with

152 genomic regions of other subspecies, suggesting they represent recombination between

153 subspecies. For the few remaining fragments showing relatively low sequence identity to all of

154 the genomes in our collection, nucleotide BLAST analysis revealed that they were more similar

155 to $M$. kansasii sequences than to any other mycobacteria, suggesting that the recombination had

156 occurred with unknown species or subspecies closely related to M. kansasii. Removing the SNVs

157 present in the recent recombinant regions significantly decreased the genetic distance between

158 strains, demonstrating the importance of recombination in the diversification of the subspecies

159 (Fig. S1).

160 The core genome alignment consists of concatenated sequences of discontinuous

161 sequence fragments in each strain, which does not fully represent the features of recombination,

162 i.e. the genomic distribution and length of recombinant fragments. Therefore, recent

163 recombination events were further explored by analysis of whole genome alignments for each of

164 the four major subspecies. Evidence of recombination was found evenly distributed across the

165 genomes of all four subspecies (Fig. S5, Fig. 3A), with fragment lengths ranging from a few base

166 pairs to a maximum of $212.9 \mathrm{~kb}$ (Fig. 2C), reminiscent of recombination by distributive

167 conjugative transfer (DCT), a form of horizontal gene transfer (HGT) in mycobacteria ${ }^{30}$.

168 In each subspecies there were both unique recombinant sequences seen in only one isolate and

169 shared recombinant sequences seen in multiple isolates, demonstrating that recombination events

170 occurred at different stages during the diversification of subspecies. For the clinically associated

171 ssp. I, the recombination donors were mainly from ssp. II and III (Fig. 2B, Fig. 3A). Fragments 
172 derived from ssp. III were more common in ssp. I strains from Europe, while ssp. I strains from

173 East Asia had recombined more frequently with ssp. II (Chi-square test, $P<0.01$ ).

\section{Genetic evidence for independent environmental acquisition of clinical infections}

After excluding the SNVs in the recombinant regions, we inferred a phylogeny for each of

177 the major subspecies to investigate the genomic differences between isolates based solely on de

178 novo mutations (Fig. 2D, Fig. 3A). Within each subspecies there were deep branches, where the

179 clinical isolates were separated by thousands of SNVs, consistent with independent infections

180 caused by unrelated environmental isolates. Besides, there were also 14 complexes, covering

181 strains in ssp. I, II, III and VI, which contained closely related isolates with an average pair-wise

182 difference less than 100 SNVs, suggesting potential dissemination of successful clones. Seven of

183 these complexes contained both isolates obtained from water and human patients, consistent with

184 the environmental strains as the source for the clinical infections. Nine of the complexes

185 contained isolates from a single geographic region, while the remaining five complexes contained

186 strains isolated on different continents.

187 The largest complex, with 268 isolates of ssp. I, was named the M. kansasii main complex

188 (MKMC). It contained 79.2\% (244/308) of all the clinical isolates included in the current study.

189 The MKMC contained 20 strains isolated from water sources, including one strain from Czech

190 that clustered with clinical strains from neighboring Poland. The remaining 19 strains were

191 isolated from an exposed cooling tower linked to a geothermal water source in Portland (a small

192 town in southeast Australia) during an outbreak of M. kansasii infections in 1990s. In the ML

193 phylogeny, these water isolates clustered with eight strains isolated from patients in the town,

194 including six patients who were part of the outbreak (Fig. 3A). The phylogenetic (median-

195 joining) network of these 27 isolates from Portland formed a star-burst structure with most

196 descendant strains surrounding the central ancestral genotype (Fig. 3B). Isolates with the

197 ancestral genotype were consistently cultured from water samples between 1990 and 1996, 
198 strongly suggesting the cooling tower was the source for this outbreak. The clinical isolates all

199 had different genotypes, and all but one were closest to the ancestral type with genomic

200 differences of 0 to 7 SNVs. The genotype of the remaining isolate was identical to a water isolate

201 and was 3 SNVs different from the ancestral type. This strongly suggests that the human

202 infections were each acquired independently from the contaminated water supply rather than by

203 human-to-human transmission. Although the outbreak had ended by 1996, after the cooling tower

204 was bypassed and the use of geothermal water discontinued, two strains belonging to the same

205 "Portland" clone were isolated from patients in 2005 and 2007 (Fig. 3B).

207 The origin and global dissemination of the M. kansasii main complex

Phylogeographic analysis of the MKMC revealed two basal branches, designated Lineage

2091 and Lineage 2. The strains from China and Australia (121/131 and 57/58 respectively)

210 predominantly belong to Lineage 2, while nearly all strains from USA and Canada were mostly

211 (20/21) belong to Lineage 1. Strains isolated in Europe were the most diverse, constituting

212 several branches in both lineages (Fig. 3A), and Bayesian phylogeographic analysis suggested

213 Europe as the most likely origin of the entire complex (Fig. S6). Several local branches contained

214 isolates exclusively from China (CHN-1, 2 and 3) or Australia (AUS-1), suggesting early

215 introductions and subsequent local expansions.

216 The isolation time of the M. kansasii strains ranged from the 1990s to 2010s, which made

217 it possible to estimate of the date of origin of the MKMC and its substitution rate using Bayesian

218 evolutionary analyses calibrated by the sampling dates. This analysis employed a subset of 121

219 ssp. I strain with short sequence reads, unambiguous collection dates and a genomic

220 recombination proportion less than $1.0 \%$, together with the reference strain ATCC 12478 isolated

221 in $1953^{31,32}$. The existence of a significant temporal signal was confirmed by both the root-to-tip

222 regression and the date randomization test (Fig. S7). Bayesian phylogenetic analysis estimated

223 the date of the MRCA of the MKMC to be around 1917 (1897-1955) (Fig. 3A, Fig. S8A) with an 
224 evolutionary rate of 1.12e-7 (95\% CI, 7.93e-8, 1.62e-7) nucleotide changes per site per year (Fig. 225 S8B).

\section{Potential gene contents contributing to the success of $M$. kansasii subspecies I}

The recent expansion of M. kansasii ssp. I and its association with clinical infections suggest that it may have evolved greater pathogenicity for human hosts than the other subspecies.

230 By comparative genomic analysis, we identified 146 genes specific to ssp. I, and several of which 231 have been associated with metabolic adaptation or virulence within human hosts (Table S2).

232 Among these are three clustered genes encoding enzymes PrpC and PrpD and regulator PrpR,

233 which are components of the methylcitrate cycle (MCC) that eliminates the toxic propionyl-CoA 234 produced during in vivo catabolism of cholesterol and fatty acids (Fig. 4A) ${ }^{33,34}$. The MCC genes 235 are located in a highly variable genomic region, and these three, along with a few flanking genes, 236 are completely or partially deleted in the other subspecies. Eighteen of the other ssp. I specific 237 genes encode potential secretory proteins of the ESX-1 system (ESP), a type VII secretion system 238 associated with virulence in M. tuberculosis ${ }^{35}$. The genes are distributed in three genomic loci, 239 one of which is comprised almost entirely of ESPs, the WhiB6 regulator and a PPE protein 240 associated with ESX-1 (Fig. 4B, Fig. S9A). Among the 18 ESPs, 10 are paralogs encoding EspE241 like proteins and all contain the WxG motif, a characteristic of ESX-1 substrates (Fig. 4C) ${ }^{36}$. A

242 BLAST search revealed that these espE-like genes are not present in the other M. kansasii

243 subspecies, nor in most other mycobacteria except $M$. marinum and closely related species such

244 as $M$. ulcerans and $M$. liflandii (Fig. S9B). In M. marinum, the espE-like genes are arranged in 245 tandem immediately upstream of the ESX-1 locus, while in M. kansasii ssp. I the three espE-like 246 containing loci are each separated from the ESX-1. Evolutionary analysis suggested that the 247 espE-like genes were independently acquired by M. marinum and M. kansasii ssp. I (Fig. S9C), 248 and then expanded in parallel (Fig. S10). 


\section{Genes under positive selection in the M. kansasii main complex}

The fixed de novo mutations (allele frequency $\geq 95 \%$ ) of all the MKMC isolates were used

252 to identify convergent mutations or genes containing an unusually high number of mutations,

253 which could be evidence of positive selection ${ }^{37,38}$. By calculating the density of nonsynonymous

254 mutations along the genome, we found that the distribution of mutations per gene followed a

255 Poisson distribution in the range of 0-3 mutations per gene (Fig. S11). However, a significant

256 deviation from this distribution was detected in 19 genes that each harbored four or more

257 mutations. Additionally, four genes were found to harbor convergent mutations that has

258 independently evolved at least three times. These 23 genes encode proteins associated with

259 diverse functions, including secondary metabolism (seven genes), DNA replication,

260 recombination and repair (four genes), metal ion transport and metabolism (three genes) and

261 carbon metabolism (three genes) (Table S3, Fig. 5).

262 The most polymorphic locus encodes Zur, the regulator of zinc uptake. A total of 36 fixed

263 zur mutations were identified in 38 clinical isolates, all of which were nonsynonymous or

264 frameshifts (Fig. 5B, Fig. S12), implying loss of function. All zur mutations could be mapped to

265 terminal nodes in the phylogeny (Fig. S12), and none was found in isolates from aquatic sources,

266 suggesting they could be adaptive mutations that emerged within the human host. Besides the

267 fixed mutations, we also identified 41 unfixed zur mutations (allele frequency $<95 \%$ ) in an

268 additional 35 clinical isolates, with several isolates carrying multiple un-linked mutations (Fig.

269 S13), strongly supporting their emergence within the host. The second most polymorphic locus

270 encodes a pair of TetR family transcriptional regulators (TetR1/2) that potentially involved in

271 Gamma-butyrolactone (GBL) signaling ${ }^{39}$. A total of 15 mutations in these two genes were found

272 in 63 isolates. This locus also exhibited highest recombination density (Fig. 5B), with evidence of

27342 independent recombinant events affecting 70 isolates. As opposed to the zur mutations, many

274 of the mutations and recombination events in the TetR1/2 locus could be mapped to inner nodes 
275 in the phylogeny (Fig. S14), suggesting that they represent potential early adaptations prior

276 infection.

The third most polymorphic locus contained two genes encoding a putative polyketide

278 synthase (Pks5) and a glucosyltransferase (WbbL2), enzymes that are involved in

279 lipooligosaccharide (LOS) synthesis ${ }^{40-42}$. A total of 14 fixed mutations distributed in 16 clinical

280 strains were detected in these two genes. Additional nonsynonymous mutations, including

281 nonsense and frameshift mutations, were detected in neighboring genes encoding other enzymes

282 associated with LOS synthesis: a fatty acyl-CoA synthetase (fadD25), an acyltransferase (papA3)

283 and two glucosyltransferases ( $g t f 1$ and $g t f 2)$ (Fig. 5C). All the fixed mutations were exclusively

284 found in clinical isolates and all but one of them could be mapped to terminal nodes in the

285 phylogeny (Fig. S12). Furthermore, unfixed mutations in those genes were identified in 18

286 clinical isolates (Table S3), suggesting they likely represent adaptive mutations emerging within

287 the host. Loss-of-function mutations in LOS biosynthesis genes have been associated with the

288 transformation from smooth to rough colony morphology in other mycobacteria ${ }^{42,43}$. Colony

289 morphology was described for 110 of the clinical strains from Shanghai (Table S1), of which 12

290 were recorded as having rough colonies. Of these 12 rough colony strains, all harbored mutations

291 in the putative LOS synthesis genes (Table S4), while none were found in the 98 smooth colony

292 strains. This high correlation between the mutations and morphology suggests that these

293 mutations affect LOS synthesis.

294 Convergent mutations that evolved at least three times were identified in the upstream of 295 three genes encoding two L-lactate dehydrogenases (lldD1, -12 C > T in three isolates; lldD2, -44

$296 \mathrm{C}>\mathrm{T}$ in six isolates) and a lipase (MKAN_RS10545, -157 G $>\mathrm{A}$ in four isolates), which involved

297 in lactate and lipid metabolisms respectively. The mutations were exclusively identified in

298 clinical isolates, and an additional of 14 clinical isolates were found harbor fixed/unfixed

299 mutations in these loci and loci nearby (Table S3). Convergent mutations were also found in the

300 coding region of $m c e 1 D$, a subunit of the Mce family transporter that is putatively responsible for 
301 lipid/sterol transportation ${ }^{44}$. Two different nucleotide substitutions were identified in codon 324

302 of $m c e 1 D$, and the mutations resulted in the same amino acid change, suggesting they were likely 303 gain-of-function mutations.

\section{Discussion}

The population genomic analyses of global M. kansasii yielded several insights into the

307 population diversity, epidemiology, evolution and pathogenicity of this important pathogen. The

308 phylogenomic analysis revealed that previously defined M. kansasii subtypes represent species

309 level lineages, as has been recently proposed ${ }^{45}$. We also found ample evidence of on-going

310 homogenous recombination between these lineages including M. gastri, but no trace of

311 recombination with any other mycobacterium species. We therefore suggest that the seven

312 lineages should be classified as M. kansasii subspecies, one of which consists of strains

313 designated as M. gastri. Extensive recent and ancestral recombination events, likely driven by

314 DCT, resulted in the mosaic genomes observed in the M. kansasii subspecies ${ }^{30}$, thereby

315 demonstrating the importance of DCT in both the subspeciation and diversification of $M$.

316 kansasii. Notably, there was evidence of recent recombination in the 16S-23S rRNA locus (Fig.

317 S3), emphasizing that species/subspecies identification should include multiple genes.

318 The comparative genomic analysis revealed genes associated with metabolism and

319 virulence that were only found in the predominant ssp. I strains, and perhaps contribute to their

320 success in colonizing and causing disease in humans. When mycobacteria infect humans, they

321 use host cholesterol and fatty acids as carbon sources, but the beta-oxidation of cholesterol and

322 odd-chain fatty acids generate propionyl-CoA that is toxic to the bacilli ${ }^{33,34,46,47}$. Pathogenic

323 mycobacteria alleviate the toxicity by consuming propionyl-CoA through the MCC cycle, which

324 is important for the growth of mycobacteria within macrophages ${ }^{34,47}$. The maintenance of the

325 MCC genes in ssp. I, and their absence in the other subspecies, may represent an adaptation to the

326 host that partially explains why this subspecies is most often associated with clinical infections. 
327 The analysis also found that ssp. I contains a special cluster of espE-like genes encoding potential

328 ESX-1 substrates that resemble the EspE, which is a highly abundant mycobacterium cell surface 329 protein secreted through the ESX-1 system ${ }^{48}$. A similar espE-like gene cluster is present in the

330 genomes of M. marinum and closely related species, and their inactivation led to defective

331 granuloma formation in zebrafish embryo ${ }^{49}$. It is therefore possible that the espE-like genes in $M$.

332 kansasii ssp. I also encode similar secretory proteins involved in modulating the host immune

333 response and causing disease. A strain of M. kansasii ssp. I was recently isolated from a river fish 334 with granulomatous nodules ${ }^{50}$, and ssp. I strain ATCC 12478 can cause chronic infection and 335 granulomas in zebrafish embryo ${ }^{51}$, suggesting that fish may be a host for ssp. I. Since $M$.

336 marinum is also known to cause infections in fish, a common host might explain the parallel

337 evolution of the espE-like gene clusters in the two species.

Isolates of ssp. I predominantly belong to a homogenous complex designated MKMC, and

339 its clinical predominance but rare isolation from city water raises the possibility of human-to-

340 human transmission. However, by investigating an outbreak of $M$. kansasii infections in

341 Australia, we found genetic evidence supporting the patients were more likely to have acquired

342 their infections independently from M. kansasii strains present in the city water supply system.

343 This is consistent with previous suggestions that city water distribution systems constitute the 344 principal reservoir for M. kansasii ${ }^{5,52}$. Our evolutionary analysis estimated that the MKMC

345 originated in the early 1900 s, possibly in Europe. Considering the association of M. kansasii

346 infections with urban areas, we speculate that the initial expansion of the MKMC was associated 347 with the rapid urbanization in Europe since the $1900 \mathrm{~s}^{53}$, and that it then spread and expanded 348 with the urbanization of other global regions. Although it is unclear how the water-born MKMC 349 could have achieved global dissemination during the past century, systems for storing potable 350 water during long voyages could have played a role.

351 Several genes involved in metabolism and the stringent response appear to be under 352 positive selections in the MKMC. Host cell lipids is a major carbon source for mycobacteria 
353 during infection and critical for the survival of bacteria within the host ${ }^{54}$. We observed

354 convergent mutations in the subunit (Mce1D) of a putative lipid/sterol transporter and the 355 upstream region of a putative lipase involved in the lipid hydrolysis ${ }^{44}$, which may represent

356 adaptations to lipid-abundant environment within the host. Besides lipids, host cell lactate was

357 recently revealed as a carbon source that is important for bacteria growth within human

358 macrophages ${ }^{55}$. More recently, convergent mutations in promoter and coding regions of lactate

359 dehydrogenase gene lldD2 were extensively identified in M. tuberculosis, and the mutations were

360 supposed to represent adaptation to changes of host ecology and were associated with higher

361 transmissibility ${ }^{56}$. We identified mutations in the upstream of $l l d D 1 / 2$ in $14 \mathrm{MKMC}$ isolates,

362 emphasizing the importance of lactate metabolism in host adaptation of mycobacteria. The

363 convergent mutations in regions upstream of $l l d D 1 / 2$ and the lipase gene likely resulted in

364 upregulation of corresponding enzymes, which may enhance the metabolic capabilities of $M$.

365 kansasii within the host and facilitate its survival and replication.

Potential positive selection was also identified in genes involved in metal ion acquisition,

367 which may represent adaptations to the limitation of metal ions (i.e., nutritional immunity) from

368 the host ${ }^{57,58}$. The highest polymorphism was found in zur, which in M. tuberculosis encodes a

369 transcriptional repressor that regulates the expression of genes involved in zinc and iron uptake

370 and zinc mobilization ${ }^{59,60}$. Inactivation of Zur could increase the expression of genes that

371 improve the ability of M. kansasii to compete with the host for the acquisition of zinc and iron

372 (Fig. S15). Two additional genes encode the iron dependent repressor (IdeR) and the subunit B of

373 the SUF system (SufB) for Fe-S cluster biosynthesis were also found under potential positive

374 selection. Mutations in these genes may augment the ability of the bacilli to maintain the iron

375 homeostasis in the iron-limited environment encountered during infection ${ }^{58,61}$ (Fig. S15).

376 Seven genes associated with secondary metabolism were found under potential positive

377 selections (Table S3), and mutations in these genes likely cause loss-of-function as the existence

378 of nonsense and/or frameshift mutations. Among these are two TetR family regulators of GBL 
signaling and two genes involved in LOS biosynthesis. GBL signaling molecules are involved in

380 the regulation of secondary metabolism and morphological development in actinomycetes ${ }^{39}$. The

381 most-studied GBL signaling is the A-factor system associated with secondary metabolism and

382 sporulation in Streptomyces ${ }^{62,63}$. Many of the mutation and recombination events in these two

383 genes mapped to inner nodes of the ML phylogeny, suggesting that they could have been

384 acquired before infecting humans, probably in city water distribution systems. While there is no

385 solid evidence of mycobacterium sporulation, inactivation of these regulators could nevertheless

386 modulate bacterial metabolism to facilitate the survival of $M$. kansasii in the urban water systems,

387 where they would encounter low levels of nutrients and disinfectant residuals. LOS are polar

388 glycolipids associated with cell wall hydrophilicity in several mycobacteria ${ }^{25,42}$. We found a high

389 correlation between loss-of-function mutations in these genes and rough colony morphology in

390 clinical isolates, consistent with previous findings in other mycobacteria ${ }^{42}$. The rough phenotype

391 resulting from the absence of LOS has been associated with an enhanced within-host survival and

392 increased virulence in several mycobacteria including M. kansasii and M. marinum ${ }^{27,64}$. In $M$.

393 tuberculosis, a recent study demonstrated that the loss of LOS occurred in its M. canettii-like

394 ancestor, which may have played a vital role in its evolution from an environmental

395 mycobacterium to a professional pathogen ${ }^{43}$. Similarly, the mutations in the LOS synthesis genes

396 of M. kansasii ssp. I could also represent in-host selection for increased virulence and the ability

397 to establish a persistent infection within the host.

The extensive selection of mutations in clinical MKMC isolates represent a feature of

399 opportunistic infections, where adaptive mutations be rapidly selected out due to the shift from

400 environment to the host niches ${ }^{65}$. Several mutations including the ones in LOS synthesis genes

401 and lldD2 promoter of the MKMC strains mimic the ancestral and/or ongoing host adaptations of

402 M. tuberculosis, which suggests a potentiality of M. kansasii to evolve to be a professional

403 pathogen. The putative adaptive mutations in the MKMC isolates were all mapped to the terminal

404 nodes in the phylogeny in this study, suggesting no transmission of the more human-adapted 
405 strains. However, considering the highly similar clinical syndromes of pulmonary infections due 406 to M. kansasii and M. tuberculosis, the possibility of aerosol transmission of M. kansasii among 407 susceptible individuals cannot be excluded, which could provide multiple rounds of host 408 adaptations for the bacteria. This should raise concern that some members of this species, 409 particularly in the MKMC, may be able to evolve into highly adapted pathogens that could persist 410 in human populations. 


\section{Material and methods}

\section{Strain collection and whole genome sequencing}

413 In China, patients with suspected pulmonary tuberculosis are referred to local designated

414 hospitals, and the positive cultures of putative NTM are subjected to species identification by $16 \mathrm{~s}$

415 rRNA sequencing in the hospitals or by a local or national branch of Center for Disease Control

416 and Prevention (CDC). Water strains of M. kansasii were isolated from public tap water across

417 Shanghai using a filtration method as previously described ${ }^{66}$. In Canada, clinical isolates were

418 obtained from the McGill University Health Centre mycobacteriology laboratory and identified

419 as M. kansasii by the Laboratoire de Sante Publique du Quebec by 16S rRNA PCR and DNA

420 sequencing. In Australia, clinical isolates were referred to the mycobacterial reference laboratory

421 at the Victorian Infectious Diseases Reference Laboratory and identified by $16 \mathrm{~S}$ rRNA PCR

422 DNA sequencing. Genomic DNA was extracted from mycobacterial cultures and sequenced on

423 either an Illumina Hiseq 2000 or an Illumina NextSeq 500 platform in single- or paired-end mode

424 with an expected depth of 100. All sequence data associated with this study was deposited in the

425 Sequence Read Archive (SRA) of NCBI under project accession PRJNA323639. Publicly

426 available genomic sequences and short read data (PRJNA317047, PRJNA374853,

427 PRJNA431546, PRJNA401515) were downloaded from the Assembly and SRA databases of

428 NCBI respectively (Supplementary Table 1).

430 Genome assembly and genomic nucleotide identity analysis

431 Sequencing reads were trimmed and filtered using Trimmomatic (v0.30) and draft genomes were

432 assembled using SPAdes (v3.13.1) in the carful mode with read correction, auto sized k-mers and 433 mismatch corrections. The quality of assembly was evaluated using Quast (v5.02) and contigs

434 less than 200bp were filtered out. Pairwise genomic ANIs were calculated using fastANI (v1.1)

435 with default parameters based on the assemblies. 


\section{Core genome analysis}

438 Core genes for all M. kansasii and M. gastri isolates included in this study were analyzed by

439 Roary (v3.11.2), through which draft genomes were first annotated using Prokka (v1.14.6) and

440 then homologous genes were clustered using the CD-Hit and MCL algorithms. To generate the

441 core-genome alignment, a minimum 90\% blastp identity, a 100\% coverage (i.e., the gene must be

442 present in all isolates) and no paralog splitting (i.e., cluster containing paralogous genes gets

443 filtered out) were set. Sequences of individual core genes were aligned with MAFFT and were

444 then concatenated into a core genome alignment according to their genomic coordinates in the

445 reference genome (NC_022663.1). RaxML (v8.2.12) was used to construct the maximum

446 likelihood phylogeny based on the core genome alignment with a GTR model and 1000 rapid

447 bootstrap replications. SplitsTree (v4.14.5) was used to construct the phylogenetic network by the

448 NeighborNet algorithm based on the core genome alignment. The Tajima $D$ statistic was

449 calculated for nonoverlapping 10-kb sliding window of the core genome alignment in each

450 subspecies using PopGenome (v2.7.5) in the R package. For identification of subspecies I

451 specific genes, a minimum $80 \%$ blastp identity with paralog splitting was set in Roary. Genes

452 present in all isolates of ssp. I but absent in all isolates of any other subspecies were selected.

454 Population structure and recombination analysis

455 Population structure was inferred using hierBASP (http://www.helsinki.fi/bsg/software/BAPS/).

456 The core genome alignment was subjected to hierBAPS analysis with a uniform prior on the

457 number of clusters. Genomic recombination was inferred using fastGEAR

458 (https://mostowylab.com/news/fastgear) based on the core genome alignment with an integration

459 number of 15 (default value). The fastGEAR used BAPS to define the "best" number of clusters

460 and then detect 'lineages' that are genetically distinct in at least 50\% of the alignment. fastGEAR

461 detects both ancestral recombination that affects all isolates in a lineage as well as recent 
462 recombination that affects a subset of isolates in a lineage. For each ancestral recombination, the

463 larger lineage was assumed to be the donor (by default).

464 The recent recombinations in isolates of the major subspecies (ssp. I, II, III and VI) were further

465 explored by using Gubbins (v2.3.4). Whole-genome alignment of each subspecies were generated

466 by an in-house pipeline based on Minimap2 (v2.17). Briefly, the contigs of individual strains

467 were aligned to the reference genome by Minimap2 with the preset parameter 'asm20' for the

468 alignment. By filtering secondary and short alignments $(<200 \mathrm{bp})$, the nucleotide corresponding

469 to the reference genome at each site is determined to generate the 'pseudogenome' for each

470 isolate. Pseudogenomes of each subspecies were concatenated to make a whole genome

471 alignment, which were subjected to Gubbins for recombination identification with default

472 parameters. The donors of the recombination fragments were determined by a BLAST search

473 against a local database containing all of the M. kansasii and M. gastri genomes included in

474 current study and the representative reference genomes for other mycobacteria collected in the

475 NCBI database. A cutoff value of identity was set to $99 \%$ to identify the probable donor strains.

476 The outputs from Gubbins were viewed with Phandango (v1.1.0), or by an in-house python script

477 that plots the recombinations with information including genomic coordinates and donor

478 subspecies.

$480 \quad$ Mapping based analysis

481 We applied mapping-based analysis to study the genetic variants among the MKMC strains. The 482 trimmed reads were mapped to the reference genome ATCC 12478 by Bowtie2 (2.3.5) and 483 variants including SNVs and short-indels were called by a SAMtools (v1.9)/VarScan (v1.4.3) 484 pipeline. Variants were called at loci where the alternative basecalls were supported by at least 485 five reads that aligned both forwardly and reversely to the reference. Variants in repeat regions, 486 putative PE/PPE family genes and transposable elements were excluded. Variants supported by $\geq$ $48795 \%$ of the mapped reads were defined as fixed/homozygous mutations, otherwise variants were 
488 defined as unfixed/heterozygous mutations. The homozygous SNVs in non-recombinant regions

489 detected by both mapping- and assembly-based analysis were used to construct the ML

490 phylogeny of the MKMC by RaxML based on the GTR model. We found several extraordinarily

491 long terminal branches in the ML phylogeny for isolates from PRJ374853, which likely represent

492 assembly errors, and the branches were thus truncated to 0. Network (v5.0) was used to generate

493 median-joining networks for the outbreak strains from Australia based on the concatenated SNV

494 sequences.

495

496 Bayesian evolutionary analysis

497 The geographic origin of the MKMC was analyzed using the Bayesian Binary MCMC (BBM)

498 method integrated in RASP (v4.0). The BBM method inputs posterior distribution of Bayesian

499 inference to reconstruct the possible ancestral distributions of given nodes via a hierarchical

500 Bayesian approach. The ML phylogeny of the MKMC constructed in the above section was used

501 for the analysis. The strains were classified into six geographic regions based on where they had

502 been isolated: East Asia, Australia, Europe, North America, South America or South Africa. The

503 Bayesian analysis was run with a fixed JC model for 5,000,000 cycles, 10 chains, a temperature

504 parameter of 0.1 , with sampling every 100 generations. Bayesian dating of the phylogeny was

505 based on a subset of 121 strains of the MKMC with short read data (to exclude assembly errors in

506 publicly available genomes), clear dates of isolation and genomes with proportions of

507 recombinations $<1.0 \%$. The reference strain ATCC 12478, which was isolated in 1953, was also

508 included. The temporal signal in the sequence alignments was investigated using TempEst

509 (v1.5.3). As a complementary assessment of the temporal signal in the data, a date randomization

510 test was performed on our datasets with the R package TipDatingBeast (v1.1-0). Sampling dates

511 of the strains were randomly shuffled 20 times, and the randomized datasets were analyzed with

512 BEAST using the same parameters as for the original datasets. If the $95 \%$ HPD intervals of root-

513 height obtained from the original data does not overlap with the estimates obtained from the 
514 randomized datasets, a statistically significant temporal structure could be confirmed. BEAST

515 (v1.8.0) software was used to determine the timescale and the evolutionary rate of the MKMC

516 using the tip-date calibration. We used an uncorrelated lognormal distribution for the substitution

517 rate, and a constant population size for the tree priors. The analysis was done in three chains of

$5185 \times 10^{7}$ generations sampled every 1,000 generations to assure independent convergence of the

519 chains. Convergence was assessed using Tracer (v1.6) to ensure that all relevant parameters

520 reached an effective population size of $>100$.

522 Detection of genes under positive selection in the MKMC

523 Genes with convergent mutations or high numbers of de novo mutations could have been subject

524 to positive selection. A subset of $247 \mathrm{MKMC}$ isolates with short reads data were used for the

525 analysis. To identify genes with multidiverse signatures, the de novo homozygous mutations of

526 all 247 MKMC isolates were used to calculate the mutation density (number of mutations per

527 gene). Under the neutral evolution model, the number of substitutions per gene is expected to

528 follow a Poisson process. The 95\% confidence interval of mean predicted values of Poissonity

529 were estimated based on the Wald interval for the mean, and a significant deviation from the

530 interval was taken as signal of potential positive selection ${ }^{37,38}$. To identify convergent mutation,

531 de novo homozygous mutations were analyzed against the maximum-likelihood phylogeny by

532 TimeTree (v0.6.4). Homoplastic mutations independently evolved at least three times were

533 identified as under potential positive selection. 
535 We thank Aina Sievers and Kathy Jackson for expert technical support. This work was supported by Natural Science Foundation of China (81661128043 and 81871625 to Q.G., 81902107 to

Q.M.), National Science and Technology Major Project of China (2017ZX10201302 and 2018ZX10715012 to Q.G., 2018ZX10103001 to Y.Z.), National Key Research and Development Program of China (No. 2017YFD0500301 to J.Y.), Sanming Project of Medicine in Shenzhen (SZSM201611030 to Q.G.), Science and Technology Department of Sichuan Province

541 (2018JY0135, to T.L.), National Health and Medical Research Council of Australia

542 (GNT1105525 to T.P.S.), CIHR Foundation Grant (FDN-148362 to M.B.), Guangdong

543 Provincial Science and Technology Program (No. 2019B030301009 to X.C.), Shenzhen Science and Technology Project (JCYJ20170412151620658, JCYJ20170307095303424 to X.C.)

\section{References}

547 1. Martin-Casabona, N. et al. Non-tuberculous mycobacteria: patterns of isolation. A multi548 country retrospective survey. Int J Tuberc Lung Dis 8, 1186-93 (2004).

549 2. Baldwin, S.L., Larsen, S.E., Ordway, D., Cassell, G. \& Coler, R.N. The complexities and challenges of preventing and treating nontuberculous mycobacterial diseases. PLoS Negl Trop Dis 13, e0007083 (2019).

3. Falkinham, J.O., 3rd. Epidemiology of infection by nontuberculous mycobacteria. Clin

554 4. Brode, S.K., Daley, C.L. \& Marras, T.K. The epidemiologic relationship between tuberculosis and non-tuberculous mycobacterial disease: a systematic review. Int J Tuberc Lung Dis 18, 1370-7 (2014).

5. Johnston, J.C., Chiang, L. \& Elwood, K. Mycobacterium kansasii. Microbiol Spectr 5(2017).

6. Wang, J. et al. Insights on the emergence of Mycobacterium tuberculosis from the analysis of Mycobacterium kansasii. Genome Biol Evol 7, 856-70 (2015).

7. Griffith, D.E. Management of disease due to Mycobacterium kansasii. Clin Chest Med 23, 613-21, vi (2002).

8. Hoefsloot, W. et al. The geographic diversity of nontuberculous mycobacteria isolated from pulmonary samples: an NTM-NET collaborative study. Eur Respir J 42, 1604-13 (2013).

9. Li, Y. et al. Mycobacterium kansasii Subtype I Is Associated With Clarithromycin Resistance in China. Front Microbiol 7, 2097 (2016). 
10. $\mathrm{Yu}, \mathrm{X}$. et al. The prevalence of non-tuberculous mycobacterial infections in mainland China: Systematic review and meta-analysis. J Infect 73, 558-567 (2016).

11. Pang, Y. et al. Diversity of nontuberculous mycobacteria in eastern and southern China: a cross-sectional study. Eur Respir J 49(2017).

12. Tan, Y. et al. Epidemiology of pulmonary disease due to nontuberculous mycobacteria in Southern China, 2013-2016. BMC Pulm Med 18, 168 (2018).

13. Wu, J. et al. Increase in nontuberculous mycobacteria isolated in Shanghai, China: results from a population-based study. PLoS One 9, e109736 (2014).

14. Vaerewijck, M.J., Huys, G., Palomino, J.C., Swings, J. \& Portaels, F. Mycobacteria in drinking water distribution systems: ecology and significance for human health. FEMS Microbiol Rev 29, 911-34 (2005).

15. Alcaide, F. et al. Heterogeneity and clonality among isolates of Mycobacterium kansasii: implications for epidemiological and pathogenicity studies. J Clin Microbiol 35, 1959-64 (1997).

16. Picardeau, M., Prod'Hom, G., Raskine, L., LePennec, M.P. \& Vincent, V. Genotypic characterization of five subspecies of Mycobacterium kansasii. J Clin Microbiol 35, 25-32 (1997).

17. Taillard, C. et al. Clinical implications of Mycobacterium kansasii species heterogeneity: Swiss National Survey. J Clin Microbiol 41, 1240-4 (2003).

18. Zhang, Y. et al. Molecular analysis of Mycobacterium kansasii isolates from the United States. J Clin Microbiol 42, 119-25 (2004).

19. Kwenda, G. et al. Molecular characterisation of clinical and environmental isolates of Mycobacterium kansasii isolates from South African gold mines. J Water Health 13, 190$202(2015)$.

20. Thomson, R., Tolson, C., Huygens, F. \& Hargreaves, M. Strain variation amongst clinical and potable water isolates of M. kansasii using automated repetitive unit PCR. Int J Med Microbiol 304, 484-9 (2014).

21. Penny, M.E., Cole, R.B. \& Gray, J. Two cases of Mycobacterium kansasii infection occurring in the same household. Tubercle 63, 129-31 (1982).

22. Ricketts, W.M., O'Shaughnessy, T.C. \& van Ingen, J. Human-to-human transmission of Mycobacterium kansasii or victims of a shared source? Eur Respir J 44, 1085-7 (2014).

23. Bryant, J.M. et al. Emergence and spread of a human-transmissible multidrug-resistant nontuberculous mycobacterium. Science 354, $751-757$ (2016).

24. Fregnan, G.B., Smith, D.W. \& Randall, H.M. Biological and chemical studies on mycobacteria. Relationship of colony morphology to mycoside content for Mycobacterium kansasil and Mycobacterium fortuitum. J Bacteriol 82, 517-27 (1961).

25. Belisle, J.T. \& Brennan, P.J. Chemical basis of rough and smooth variation in mycobacteria. J Bacteriol 171, 3465-70 (1989).

26. Pawlik, A. et al. Identification and characterization of the genetic changes responsible for the characteristic smooth-to-rough morphotype alterations of clinically persistent Mycobacterium abscessus. Mol Microbiol 90, 612-29 (2013).

27. Collins, F.M. \& Cunningham, D.S. Systemic Mycobacterium kansasii infection and regulation of the alloantigenic response. Infect Immun 32, 614-24 (1981). 
28. Konstantinidis, K.T. \& Tiedje, J.M. Genomic insights that advance the species definition for prokaryotes. Proc Natl Acad Sci US A 102, 2567-72 (2005).

29. Jain, C., Rodriguez, R.L., Phillippy, A.M., Konstantinidis, K.T. \& Aluru, S. High throughput ANI analysis of $90 \mathrm{~K}$ prokaryotic genomes reveals clear species boundaries. Nat Commun 9, 5114 (2018).

30. Gray, T.A. \& Derbyshire, K.M. Blending genomes: distributive conjugal transfer in mycobacteria, a sexier form of HGT. Mol Microbiol 108, 601-613 (2018).

31. Buhler, V.B. \& Pollak, A. Human infection with atypical acid-fast organisms; report of two cases with pathologic findings. Am J Clin Pathol 23, 363-74 (1953).

32. LESSEL, E.F. Bacterial type cultures of the American Type Culture Collection. I. International Journal of Systematic and Evolutionary Microbiology 12, 71-88 (1962).

33. Dolan, S.K. et al. Loving the poison: the methylcitrate cycle and bacterial pathogenesis. Microbiology 164, 251-259 (2018).

34. Griffin, J.E. et al. Cholesterol catabolism by Mycobacterium tuberculosis requires transcriptional and metabolic adaptations. Chem Biol 19, 218-27 (2012).

35. Groschel, M.I., Sayes, F., Simeone, R., Majlessi, L. \& Brosch, R. ESX secretion systems: mycobacterial evolution to counter host immunity. Nat Rev Microbiol 14, 677-691 (2016).

36. Houben, E.N., Korotkov, K.V. \& Bitter, W. Take five - Type VII secretion systems of Mycobacteria. Biochim Biophys Acta 1843, 1707-16 (2014).

37. Hedge, J. \& Wilson, D.J. Practical Approaches for Detecting Selection in Microbial Genomes. PLoS Comput Biol 12, e1004739 (2016).

38. Holt, K.E. et al. High-throughput sequencing provides insights into genome variation and evolution in Salmonella Typhi. Nat Genet 40, 987-93 (2008).

39. Cuthbertson, L. \& Nodwell, J.R. The TetR family of regulators. Microbiol Mol Biol Rev 77, 440-75 (2013).

40. Etienne, G. et al. Identification of the polyketide synthase involved in the biosynthesis of the surface-exposed lipooligosaccharides in mycobacteria. J Bacteriol 191, 2613-21 (2009).

41. Nataraj, V. et al. MKAN27435 is required for the biosynthesis of higher subclasses of lipooligosaccharides in Mycobacterium kansasii. PLoS One 10, e0122804 (2015).

42. van der Woude, A.D. et al. Unexpected link between lipooligosaccharide biosynthesis and surface protein release in Mycobacterium marinum. J Biol Chem 287, 20417-29 (2012).

43. Boritsch, E.C. et al. pks5-recombination-mediated surface remodelling in Mycobacterium tuberculosis emergence. Nat Microbiol 1, 15019 (2016).

44. Casali, N. \& Riley, L.W. A phylogenomic analysis of the Actinomycetales mce operons. BMC Genomics 8, 60 (2007).

45. Tagini, F. et al. Phylogenomics reveal that Mycobacterium kansasii subtypes are specieslevel lineages. Description of Mycobacterium pseudokansasii sp. nov., Mycobacterium innocens sp. nov. and Mycobacterium attenuatum sp. nov. Int J Syst Evol Microbiol 69, 1696-1704 (2019).

46. Eoh, H. \& Rhee, K.Y. Methylcitrate cycle defines the bactericidal essentiality of isocitrate lyase for survival of Mycobacterium tuberculosis on fatty acids. Proc Natl Acad Sci US A 111, 4976-81 (2014). 
47. Munoz-Elias, E.J., Upton, A.M., Cherian, J. \& McKinney, J.D. Role of the methylcitrate cycle in Mycobacterium tuberculosis metabolism, intracellular growth, and virulence. $\mathrm{Mol}$ Microbiol 60, 1109-22 (2006).

48. Sani, M. et al. Direct visualization by cryo-EM of the mycobacterial capsular layer: a labile structure containing ESX-1-secreted proteins. PLoS Pathog 6, e1000794 (2010).

49. Stoop, E.J. et al. Zebrafish embryo screen for mycobacterial genes involved in the initiation of granuloma formation reveals a newly identified ESX-1 component. Dis Model Mech 4, 526-36 (2011).

50. Terrazas, M.M., Bradway, D.S., Staigmiller, K.D., Wipf, M.M. \& Snekvik, K. Identification of Mycobacterium kansasii and a Mycobacterium sp. in Salmonids from the Missouri River, Montana. Northwestern Naturalist 97, 98-104 (2016).

51. Johansen, M.D. \& Kremer, L. A zebrafish model of Mycobacterium kansasii infection reveals large extracellular cord formation. J Infect Dis (2020).

52. Ahn, C.H., Lowell, J.R., Onstad, G.D., Shuford, E.H. \& Hurst, G.A. A demographic study of disease due to Mycobacterium kansasii or M intracellulare-avium in Texas. Chest 75, 120-5 (1979).

53. Bairoch, P. \& Goertz, G. Factors of Urbanisation in the Nineteenth Century Developed Countries:A Descriptive and Econometric Analysis. Urban Studies 23, 285-305 (1986).

54. Singh, G., Singh, G., Jadeja, D. \& Kaur, J. Lipid hydrolizing enzymes in virulence: Mycobacterium tuberculosis as a model system. Crit Rev Microbiol 36, 259-69 (2010).

55. Billig, S. et al. Lactate oxidation facilitates growth of Mycobacterium tuberculosis in human macrophages. Sci Rep 7, 6484 (2017).

56. Brynildsrud, O.B. et al. Global expansion of Mycobacterium tuberculosis lineage 4 shaped by colonial migration and local adaptation. Sci Adv 4, eaat5869 (2018).

57. Li, Y. et al. Zinc depletion induces ribosome hibernation in mycobacteria. Proc Natl Acad Sci U S A 115, 8191-8196 (2018).

58. Sritharan, M. Iron Homeostasis in Mycobacterium tuberculosis: Mechanistic Insights into Siderophore-Mediated Iron Uptake. J Bacteriol 198, 2399-409 (2016).

59. Maciag, A. et al. Global analysis of the Mycobacterium tuberculosis Zur (FurB) regulon. J Bacteriol 189, 730-40 (2007).

60. Riccardi, G., Milano, A., Pasca, M.R. \& Nies, D.H. Genomic analysis of zinc homeostasis in Mycobacterium tuberculosis. FEMS Microbiol Lett 287, 1-7 (2008).

61. Pandey, M., Talwar, S., Bose, S. \& Pandey, A.K. Iron homeostasis in Mycobacterium tuberculosis is essential for persistence. Sci Rep 8, 17359 (2018).

62. Miyake, K., Kuzuyama, T., Horinouchi, S. \& Beppu, T. The A-factor-binding protein of Streptomyces griseus negatively controls streptomycin production and sporulation. $J$ Bacteriol 172, 3003-8 (1990).

63. Onaka, H., Nakagawa, T. \& Horinouchi, S. Involvement of two A-factor receptor homologues in Streptomyces coelicolor A3(2) in the regulation of secondary metabolism and morphogenesis. Mol Microbiol 28, 743-53 (1998).

64. Alibaud, L. et al. Increased phagocytosis of Mycobacterium marinum mutants defective in lipooligosaccharide production: a structure-activity relationship study. J Biol Chem 289, 215-28 (2014). 
698 65. Didelot, X., Walker, A.S., Peto, T.E., Crook, D.W. \& Wilson, D.J. Within-host evolution 699 of bacterial pathogens. Nat Rev Microbiol 14, 150-62 (2016).

700 66. Le Dantec, C. et al. Occurrence of mycobacteria in water treatment lines and in water distribution systems. Appl Environ Microbiol 68, 5318-25 (2002). 


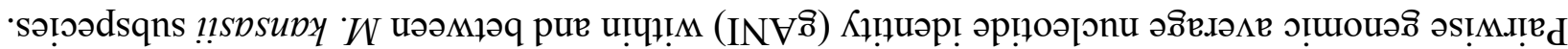

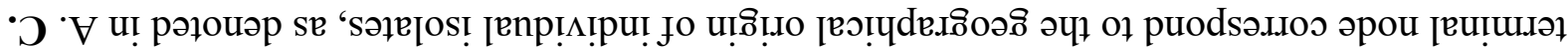

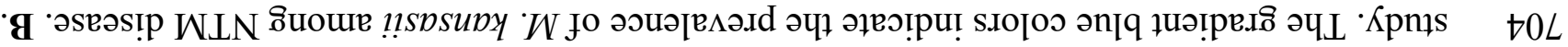

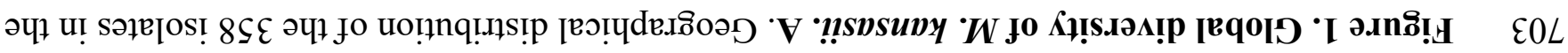

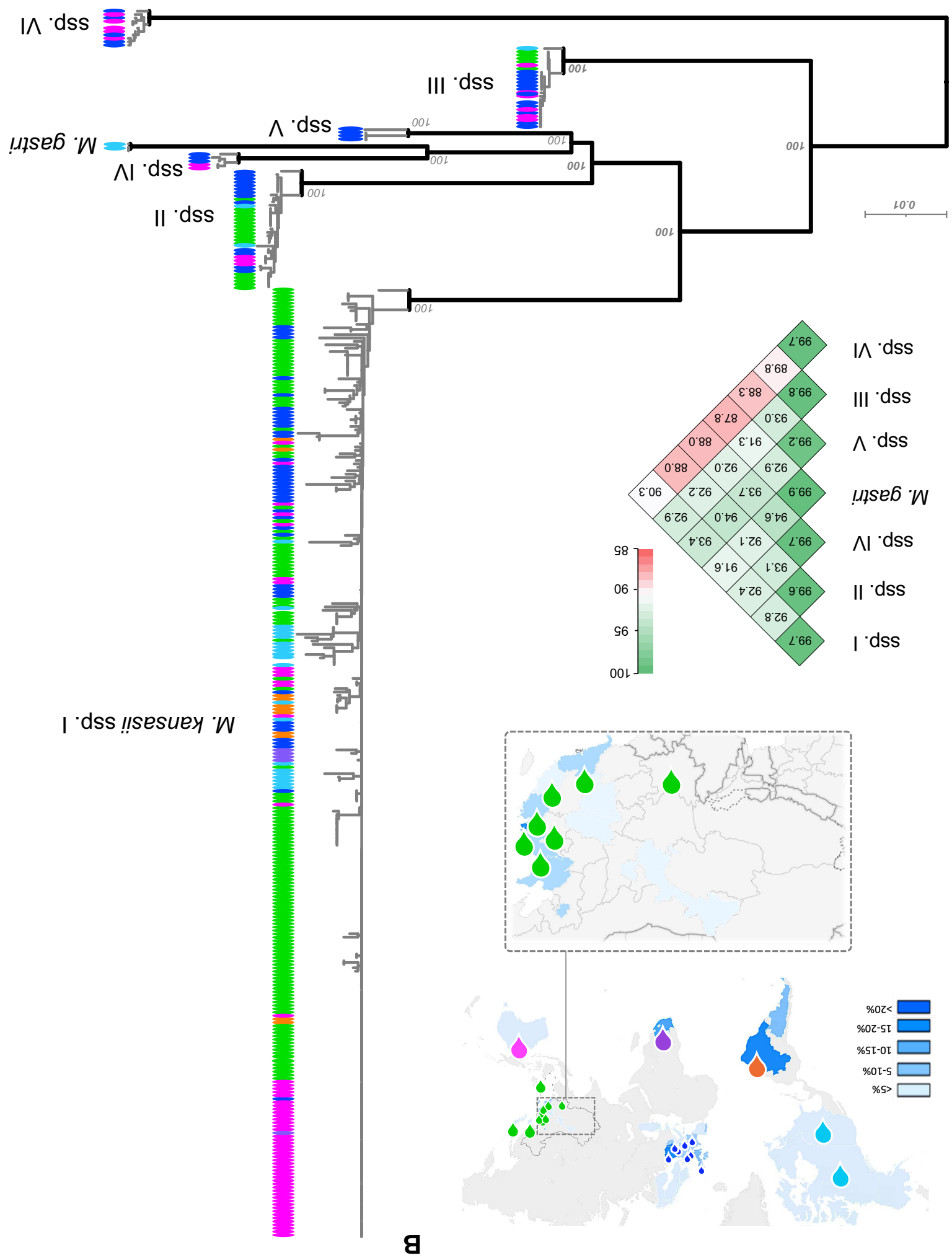

Z0L 
A

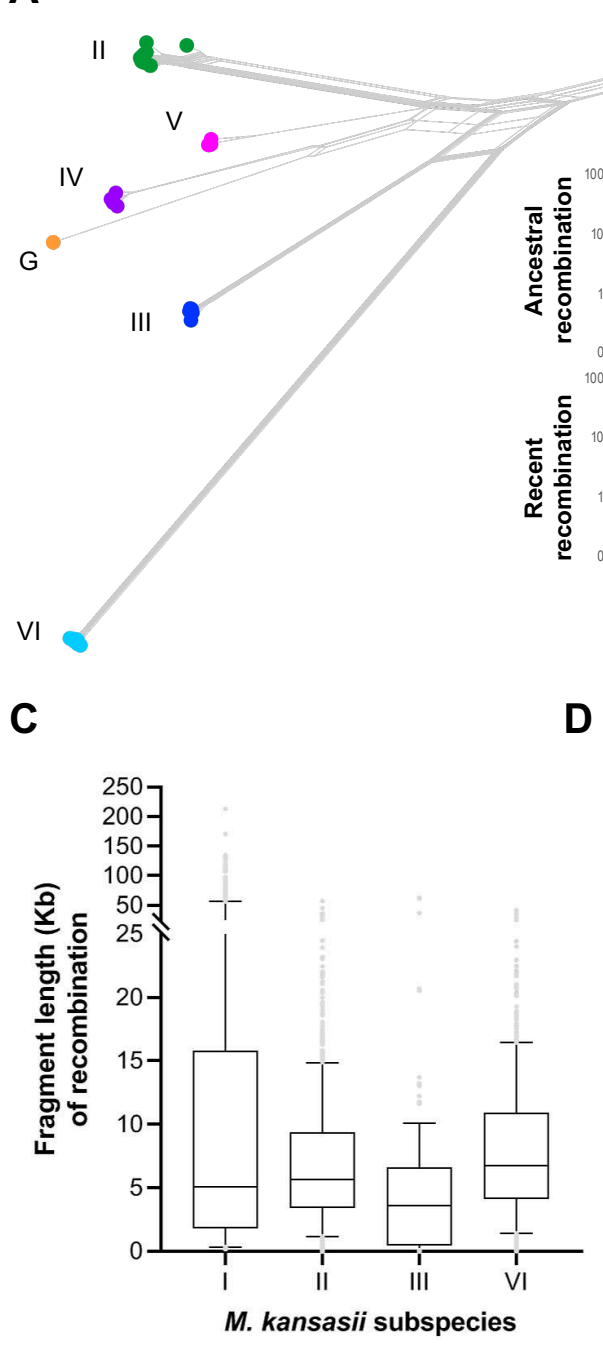

D
B
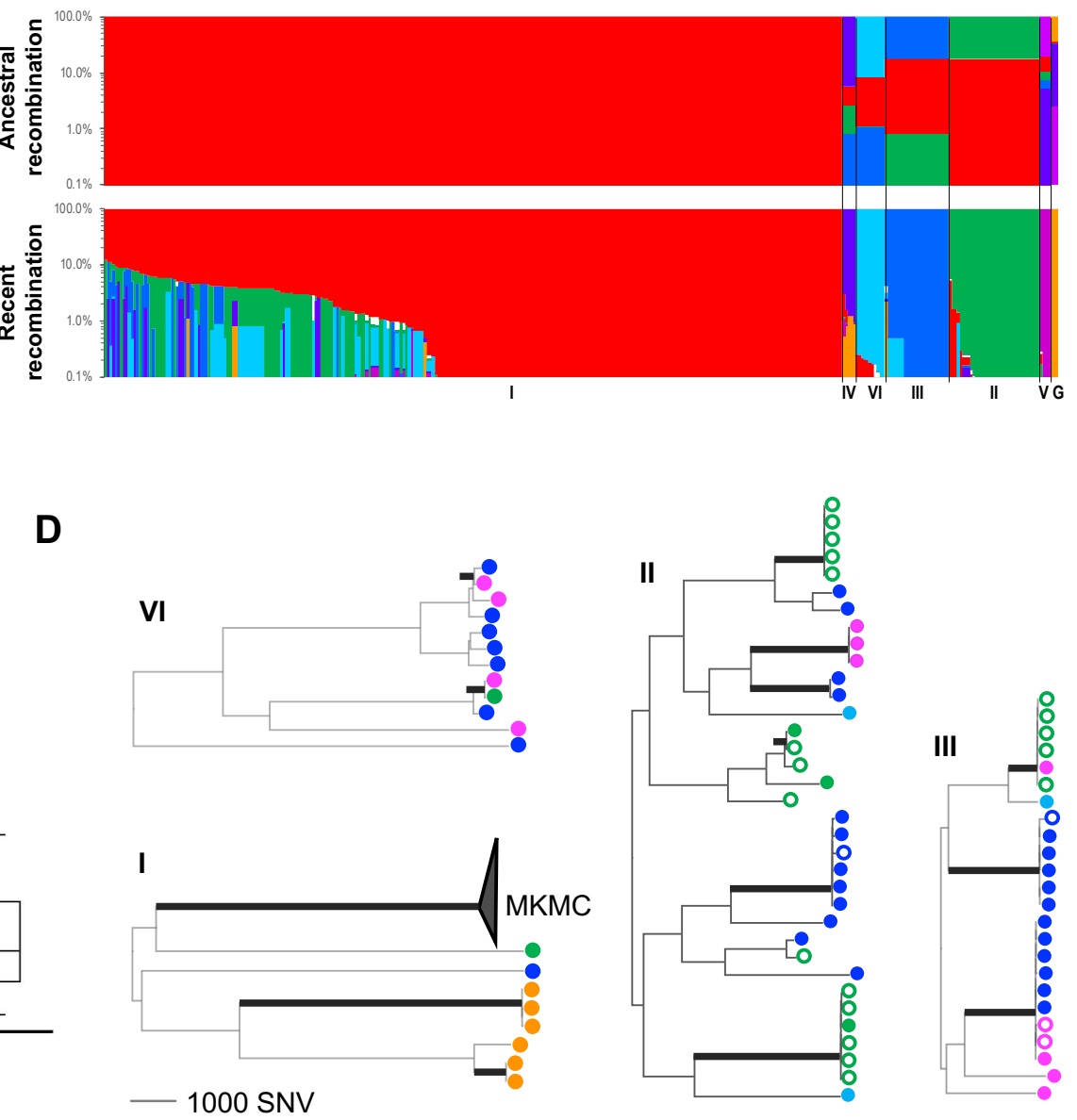
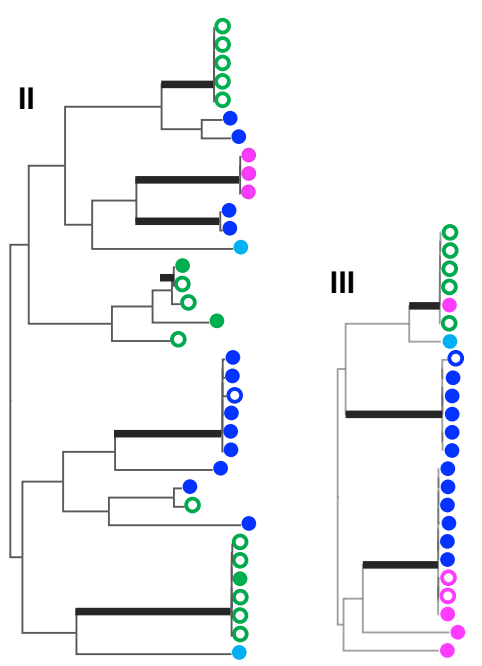

Figure 2. Genomic recombination and its contribution in subspeciation and diversification

712 alignment of 358 isolates. I-VI, M. kansasii subspecies; G, M. gastri. B. Population structure and

713 genomic recombinations inferred by fastGEAR. Each line represents the genomic constitution

714 (exhibited as color strips) of individual isolates according to ancestral (upper panel) or recent

715 (lower panel) recombinations. Colors represent the different subspecies corresponding to panel A.

716 Strips in white color (lower section) represent recent recombinations from unknown sources. C.

717 Length distribution of recombinant fragments in the four major subspecies. The box plots

718 indicate the 5-95\% percentiles and quartiles. D. Maximum likelihood phylogeny of the four

719 major subspecies based on de novo SNVs in non-recombinant regions. Branches highlighted in

720 bold indicate homogenous complexes containing isolates with an averaged pair-wise genomic

721 difference less than 100 SNVs. Colors of terminal nodes indicate geographical origins

722 corresponding to Figure 1. Filed circles indicate a human source; empty circles, an environmental

723 source. 
A

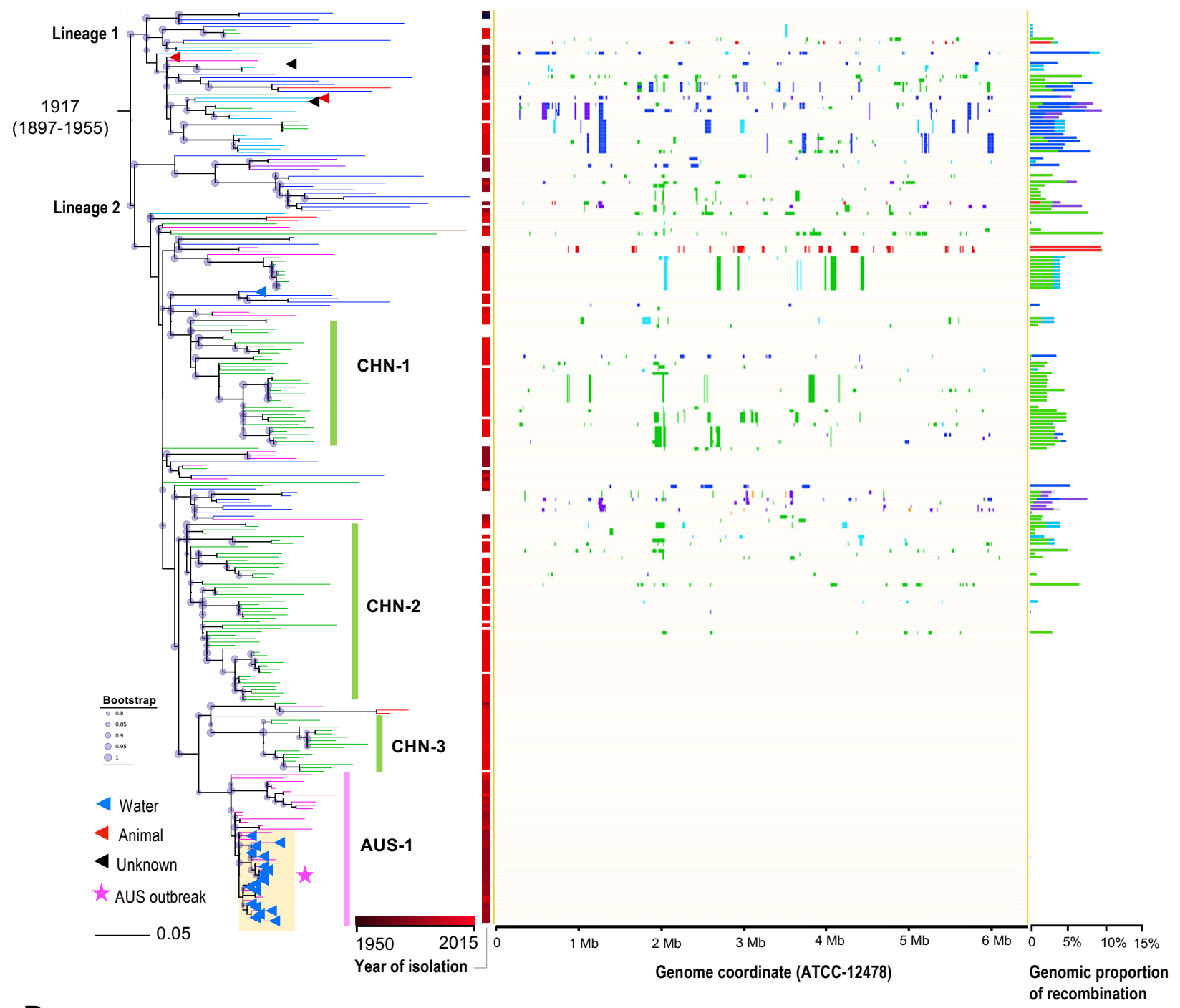

B

Figure 3. Phylogenomic analyses of the M. kansasii main complex (MKMC). A. Left panel, the maximum-likelihood phylogeny of the MKMC based on de novo mutations. Isolation from non-human or unknown sources are indicated by triangles in the terminal nodes. Right panel, genomic pattern and proportion of recent recombinations for individual isolates. Donor

729 subspecies are colored corresponding to Figure 2. B. Median-Joining network for the Australia

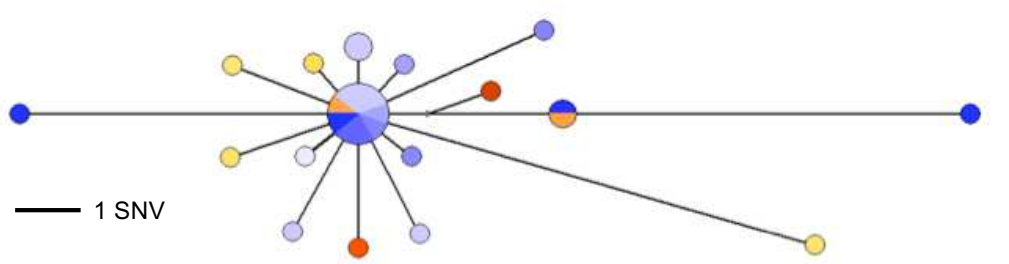

outbreak strain cluster. Node size, number of isolates; node color, source and year of isolation. 
A

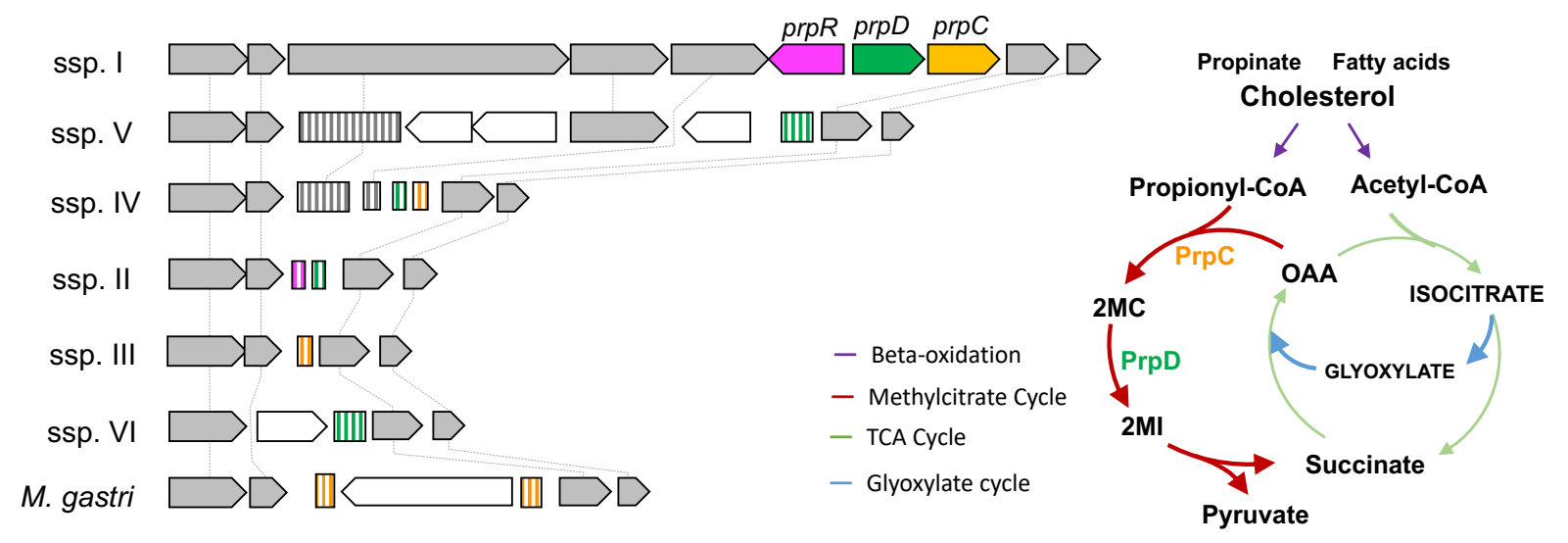

B

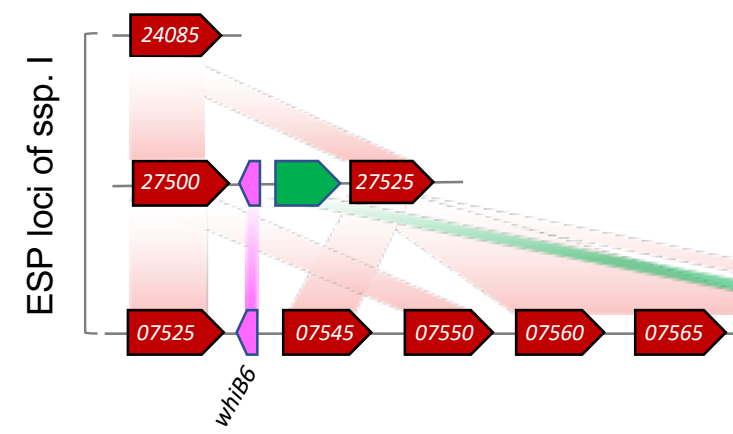

C

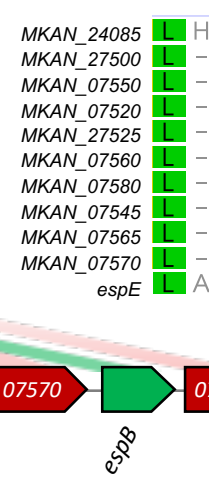

100 $\begin{array}{llllllllll}A & P & D & G & G & W & Q & G\end{array}$

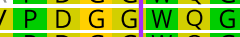
P D G G W Q \begin{tabular}{llllllll}
$V$ & $P$ & $D$ & $S$ & $G$ & $W$ & $Q$ & $G$ \\
\hline
\end{tabular} \begin{tabular}{llllllll}
$V$ & $P$ & $G$ & $G$ & $G$ & $W$ & $Q$ & $G$ \\
\hline
\end{tabular} \begin{tabular}{lllll|lll}
$V$ & $P$ & $D$ & $G$ & $G$ & $W$ & $Q$ & $G$ \\
$A$ & $P$ & $D$ & $G$ & $G$ & $W$ & $Q$ & $G$
\end{tabular} \begin{tabular}{lllll|lll}
$A$ & $P$ & $D$ & $G$ & $G$ & $W$ & $Q$ & $G$ \\
\hline & $P$ & $D$ & $D$ & $G$ & $W$ & $Q$ & $G$
\end{tabular} \begin{tabular}{llllllll}
$V$ & $P$ & $D$ & $G$ & $G$ & $W$ & $Q$ & $G$ \\
\hline
\end{tabular} V P D G GW Q G I P G T NW I G

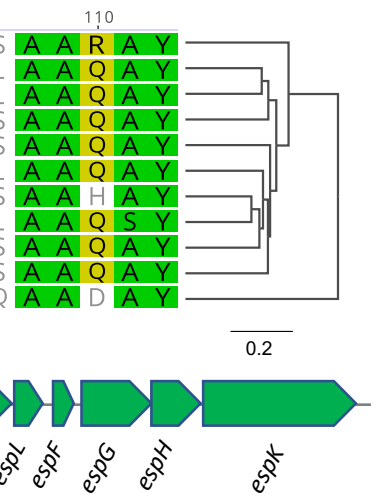

732 Figure 4. Genomic loci specific to M. kansasii ssp. I. A. Left, synteny map for the genomic

733 region flanking the MCC genes in M. kansasii subspecies. Full-length and truncated genes are

734 represented by arrows and rectangles respectively. The full MCC genes in ssp. I and their

735 orthologous sequences in the other subspecies are indicated with different colors. The flanking

736 genes are in gray or white to represent homologous or orphan genes, respectively. Right, a

737 scheme of the methylcitrate cycle of mycobacteria and its relation with the beta-oxidation, TCA

738 and glyoxylate cycles. B. Synteny map of the three ESP loci specific to M. kansasii ssp. I. C.

739 Sequence similarity between the EspE and the EspE-like proteins of M. kansasii ssp. I. 
A

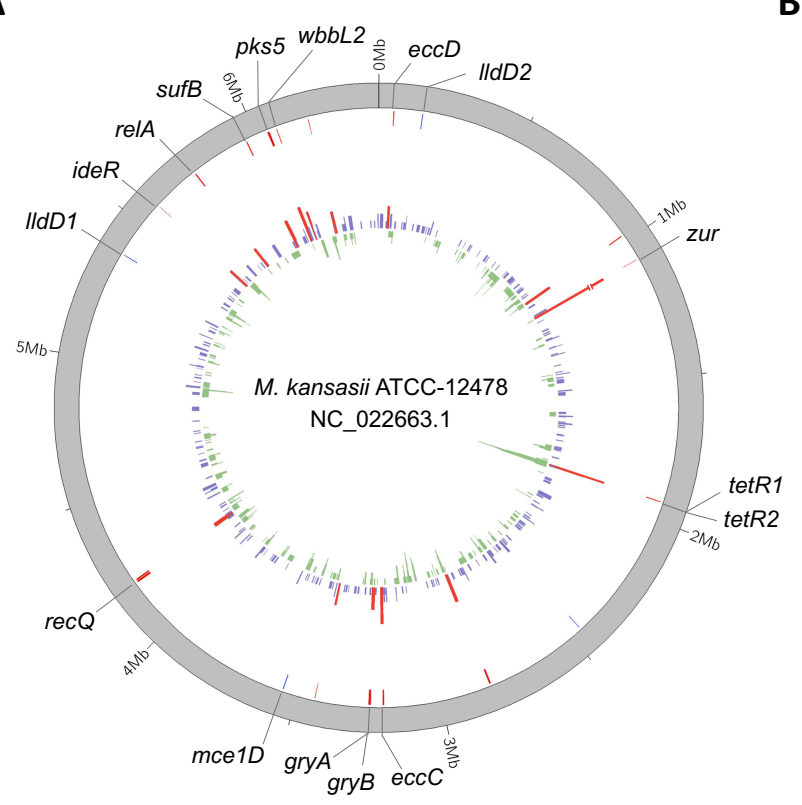

B
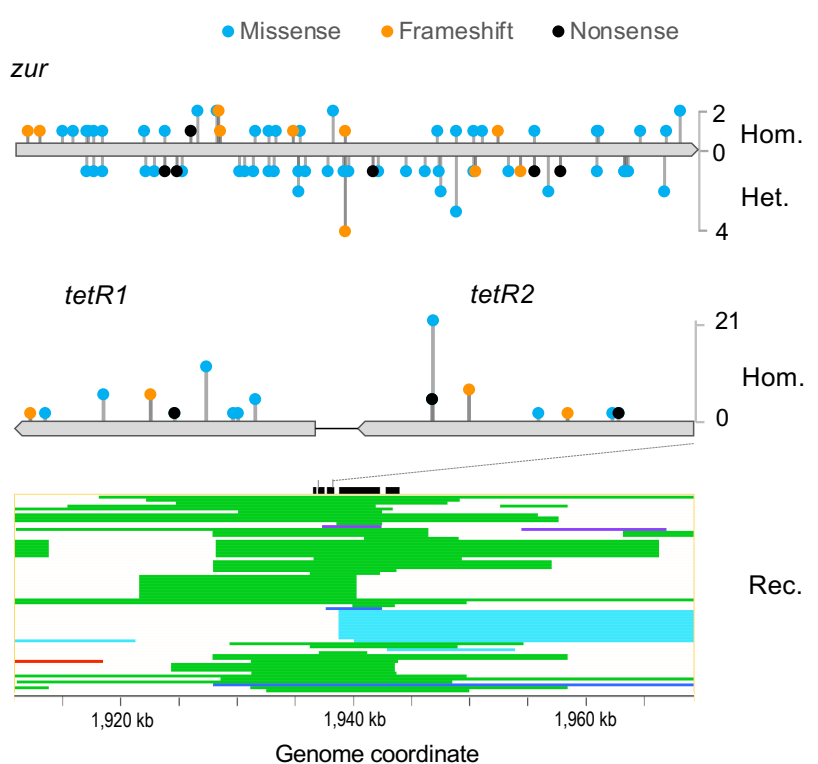

C

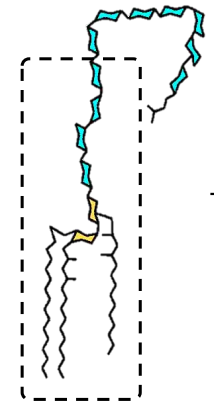

LOS

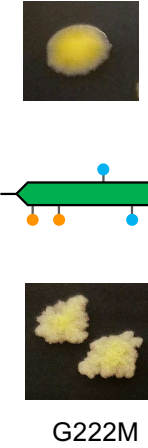

Wild-type

Figure 5. Genes under positive selections in the MKMC. A. Circular plot of genes under potential positive selection. Innermost inward bars, number of recombination per gene; innermost outward bars, number of mutations per gene (red bars, $n \geq 4$ ); outer red lines, location of highly polymorphic genes; outer blue line, location of genes with convergent mutations. B. Schematic diagrams depicting the distribution and frequency of non-synonymous mutations in zur and tet $R 1 / 2$, and the recombinations around tetR $1 / 2$. Hom., homozygous mutation; Het., heterozygous mutation; Rec., recombination (strip colors represent donor subspecies corresponding to Figure 2). C. Schematic representation of mutations in LOS biosynthesis genes and corresponding morphology of the mutant strains. Genes were colored according to their functions. Green, genes involved in polyketide synthesis; orange, acyltransferase; cyan, glucosyltransferase. 
Figures

A

C
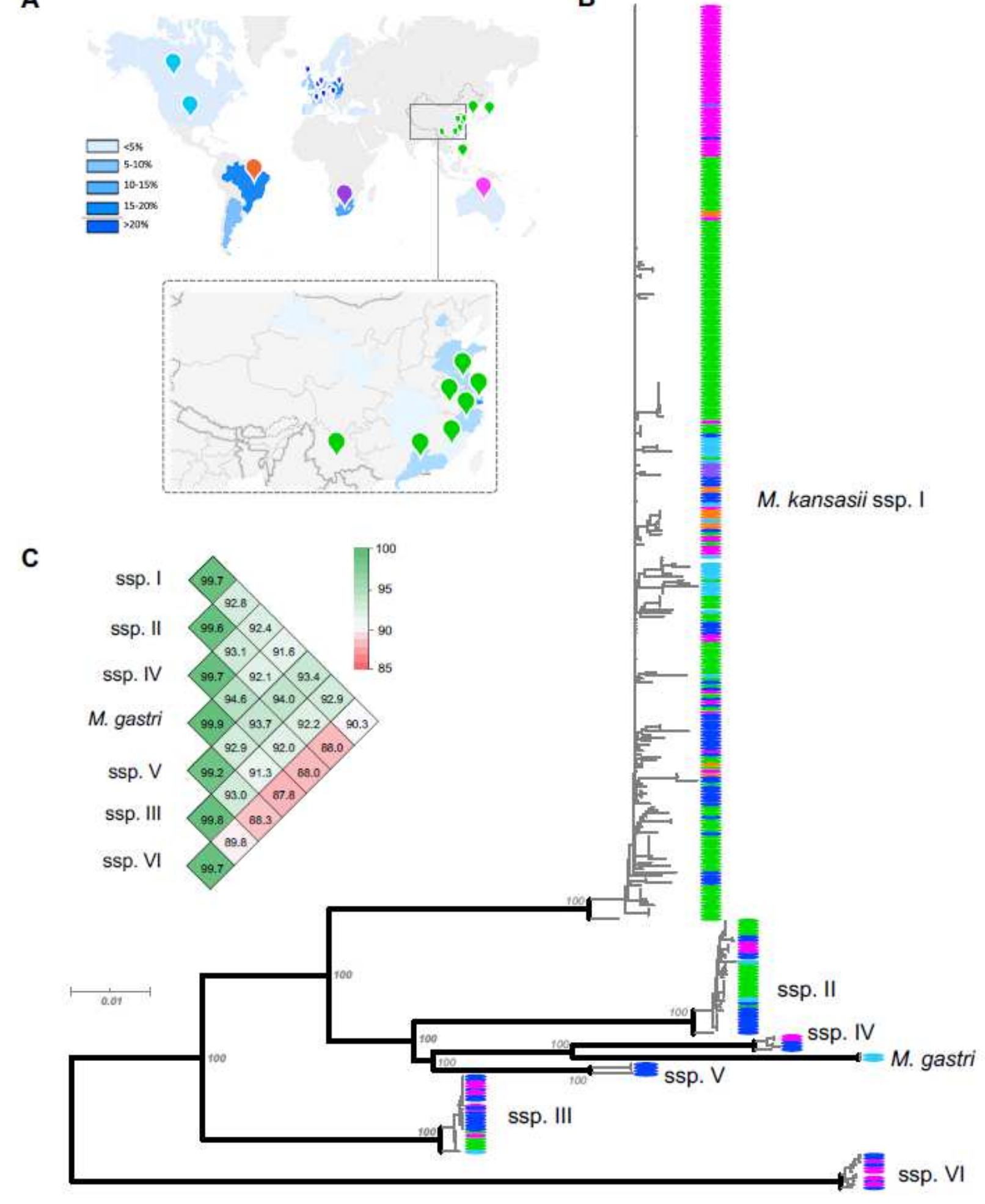

B

Figure 1

Global diversity of M. kansasii. A. Geographical distribution of the 358 isolates in the study. The gradient blue colors indicate the prevalence of M. kansasii among NTM disease. B. Core genome based maximum-likelihood phylogeny of the 358 isolates. The colors of the terminal node correspond to the 
geographical origin of individual isolates, as denoted in A. C. Pairwise genomic average nucleotide identity (gANI) within and between M. kansasii subspecies.

A

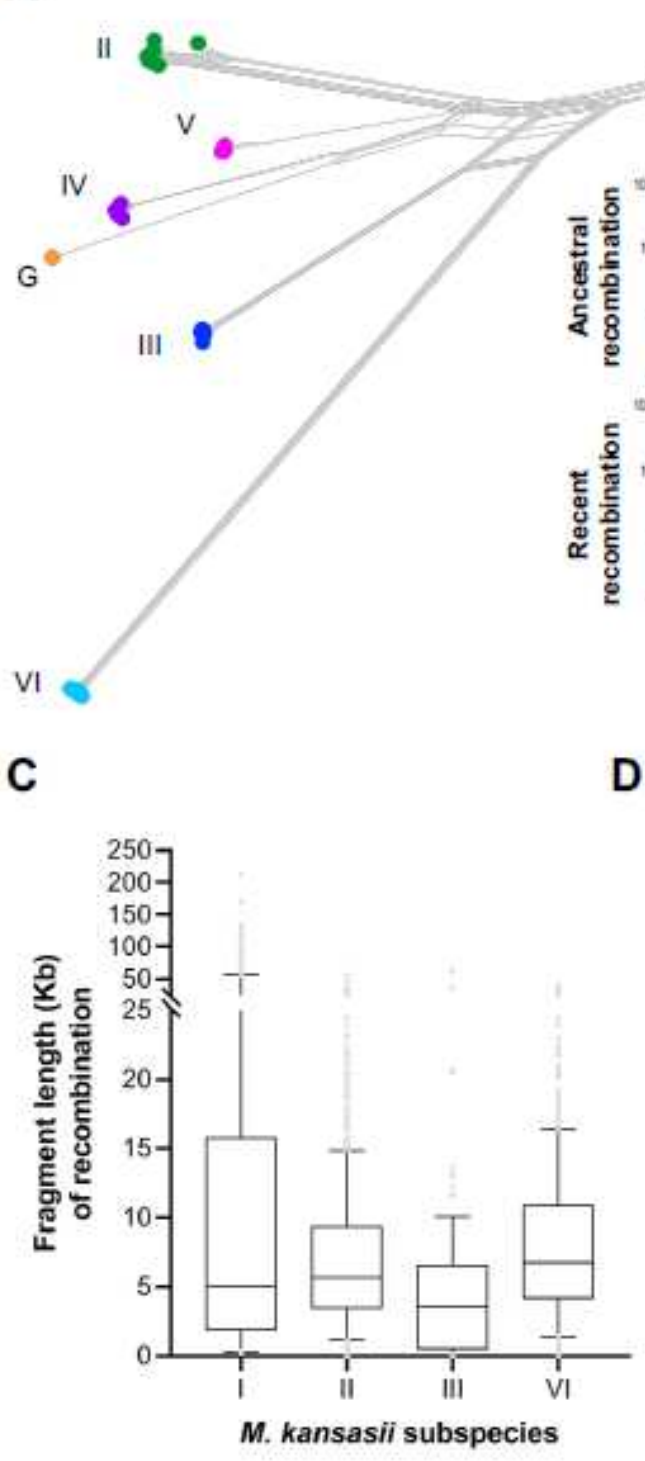

B

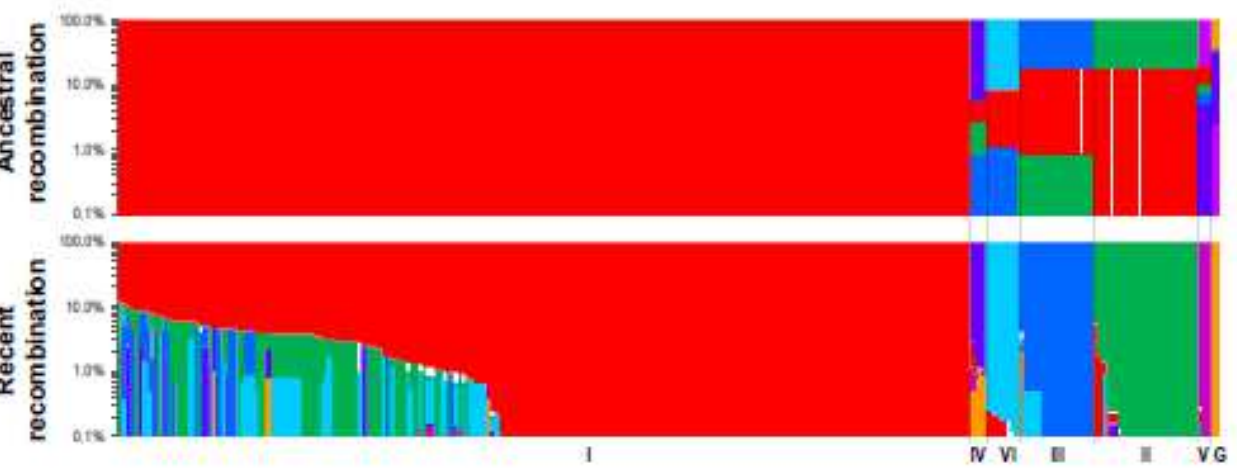

D
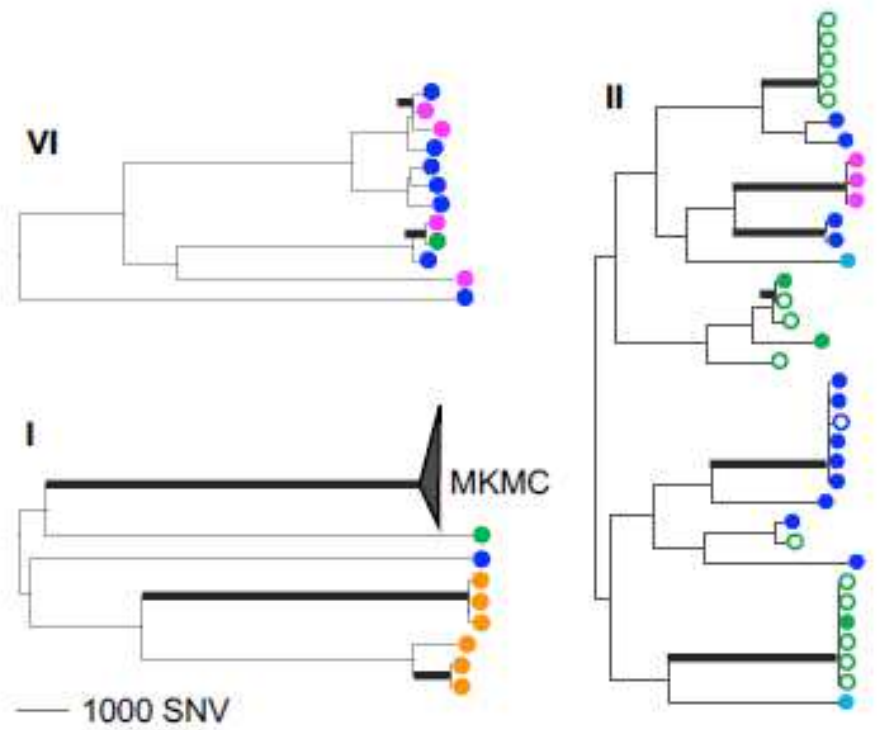

\section{Figure 2}

Genomic recombination and its contribution in subspeciation and diversification of M. kansasii. A. Phylogenetic network of $\mathrm{M}$. kansasii subspecies based on the core genome alignment of 358 isolates. IVI, M. kansasii subspecies; G, M. gastri. B. Population structure and genomic recombinations inferred by fastGEAR. Each line represents the genomic constitution (exhibited as color strips) of individual isolates according to ancestral (upper panel) or recent (lower panel) recombinations. Colors represent the different subspecies corresponding to panel A. Strips in white color (lower section) represent recent recombinations from unknown sources. C. Length distribution of recombinant fragments in the four major subspecies. The box plots indicate the 5-95\% percentiles and quartiles. D. Maximum likelihood phylogeny of the four major subspecies based on de novo SNVs in non-recombinant regions. Branches highlighted in bold indicate homogenous complexes containing isolates with an averaged pair-wise 
genomic difference less than 100 SNVs. Colors of terminal nodes indicate geographical origins corresponding to Figure 1. Filed circles indicate a human source; empty circles, an environmental source.

A

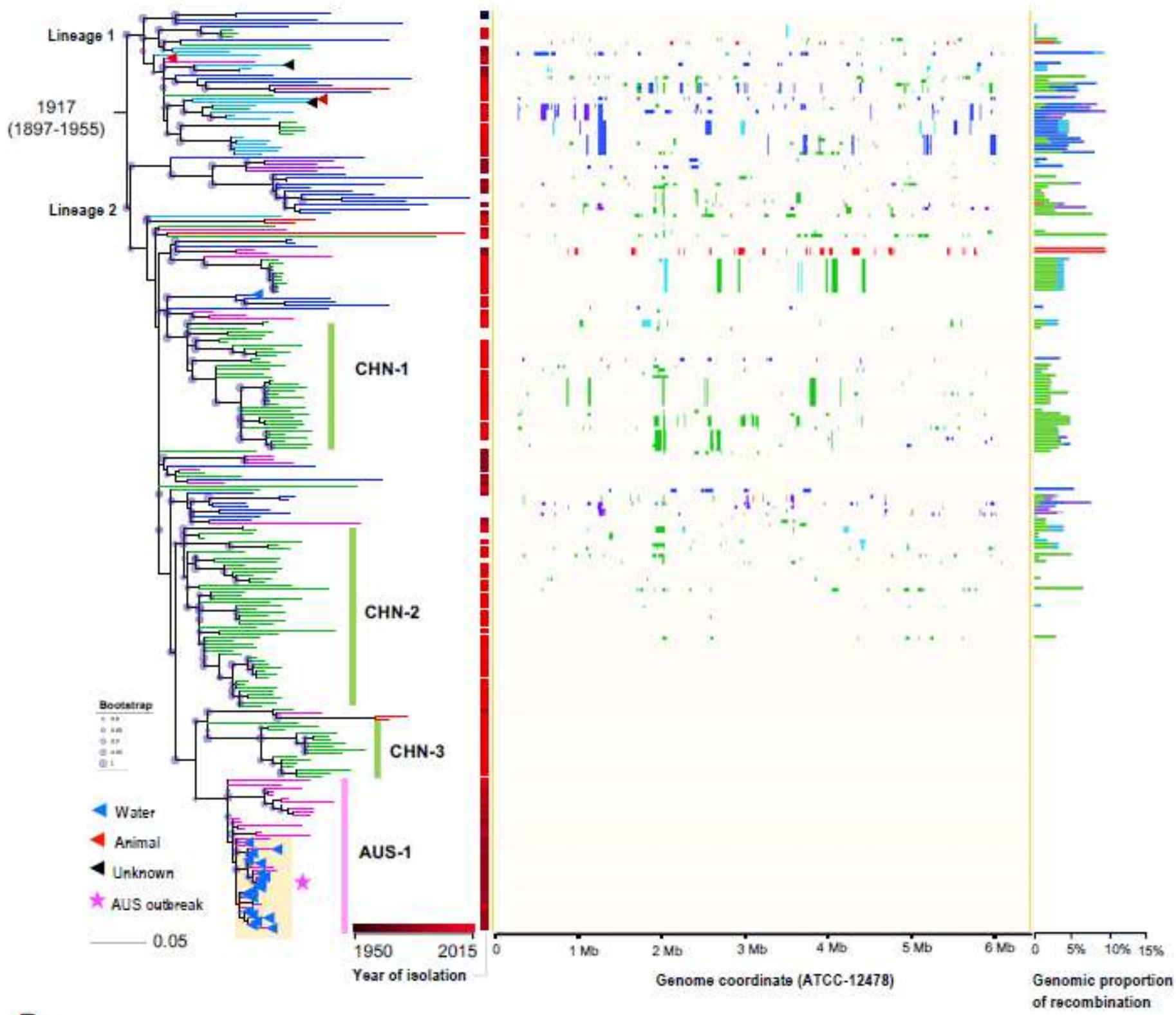

B
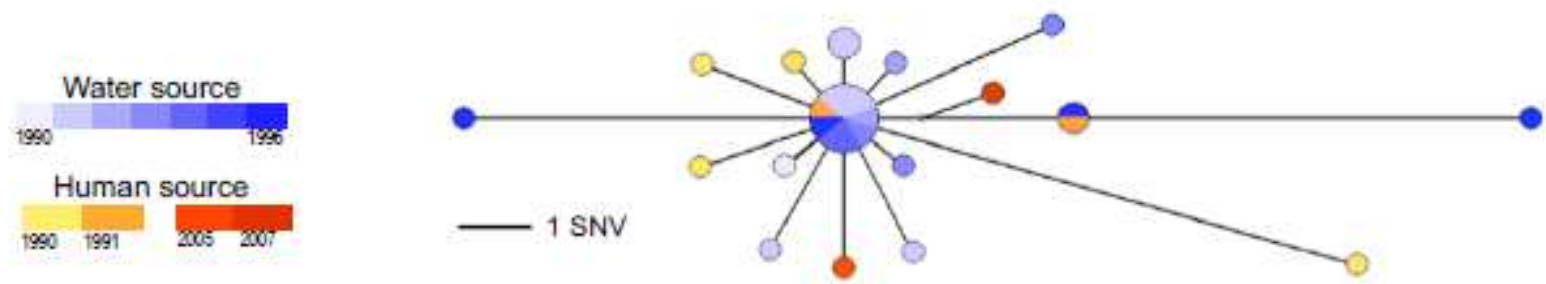

Figure 3

Phylogenomic analyses of the M. kansasii main complex (MKMC). A. Left panel, the maximum-likelihood phylogeny of the MKMC based on de novo mutations. Isolation from non-human or unknown sources are indicated by triangles in the terminal nodes. Right panel, genomic pattern and proportion of recent 
recombinations for individual isolates. Donor subspecies are colored corresponding to Figure 2. B. Median-Joining network for the Australia outbreak strain cluster. Node size, number of isolates; node color, source and year of isolation.

A

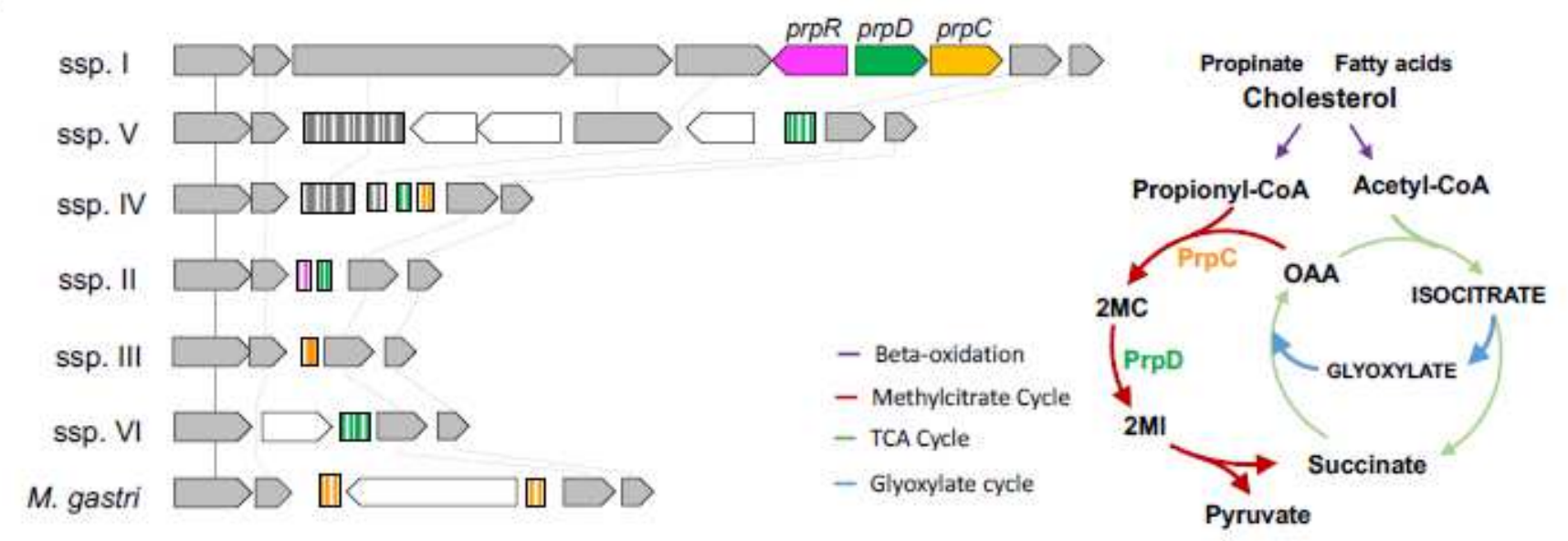

B

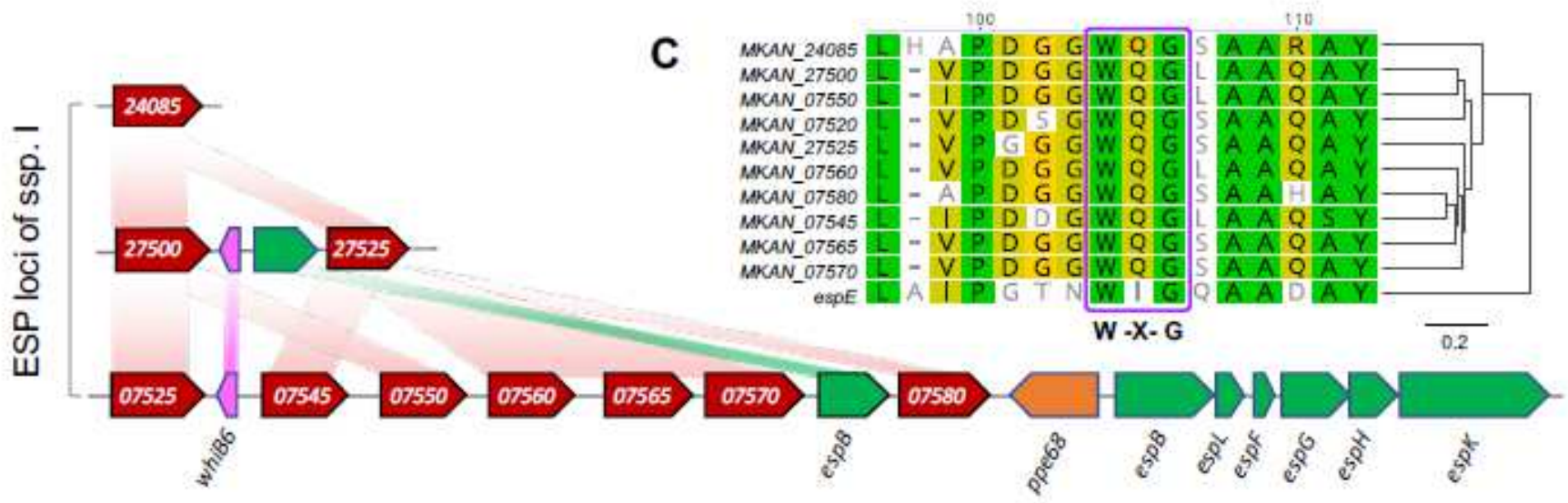

\section{Figure 4}

Genomic loci specific to M. kansasii ssp. I. A. Left, synteny map for the genomic region flanking the MCC genes in M. kansasii subspecies. Full-length and truncated genes are represented by arrows and rectangles respectively. The full MCC genes in ssp. I and their orthologous sequences in the other subspecies are indicated with different colors. The flanking genes are in gray or white to represent homologous or orphan genes, respectively. Right, a scheme of the methylcitrate cycle of mycobacteria and its relation with the beta-oxidation, TCA and glyoxylate cycles. B. Synteny map of the three ESP loci specific to M. kansasii ssp. I. C. Sequence similarity between the EspE and the EspE-like proteins of M. kansasii ssp. I. 
A

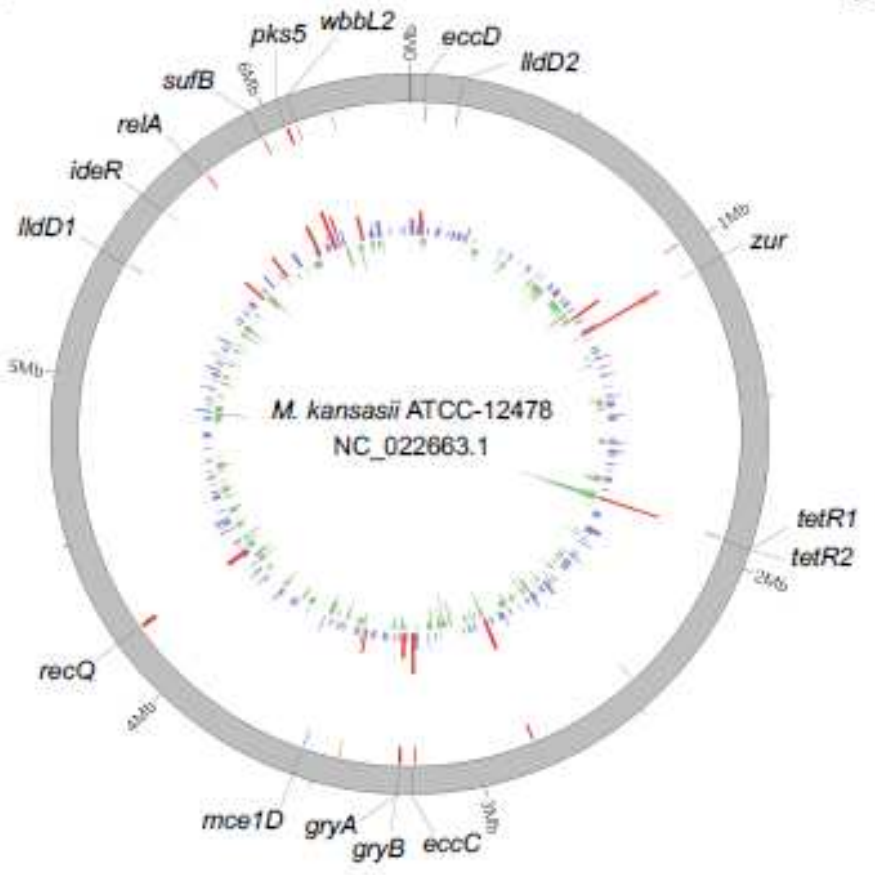

C

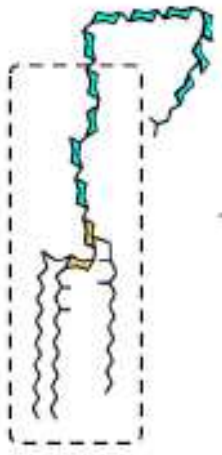

LOS

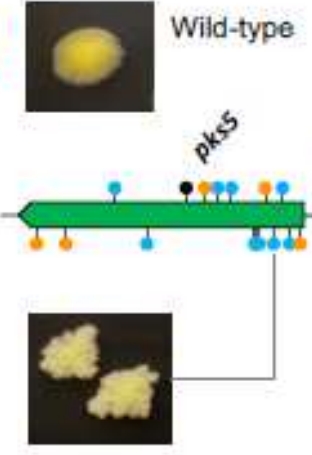

G222M

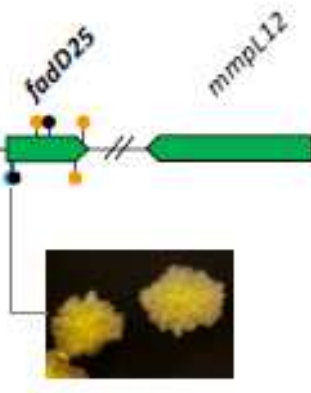

S7A, L8S

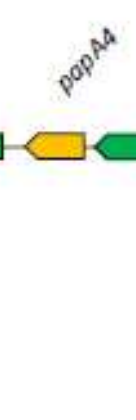

- Missense * Frameshift - Nonsense

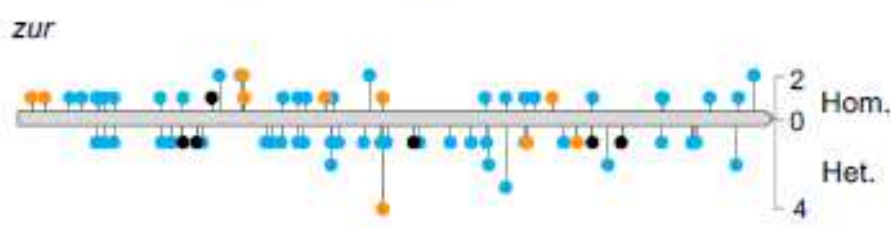

tetR1

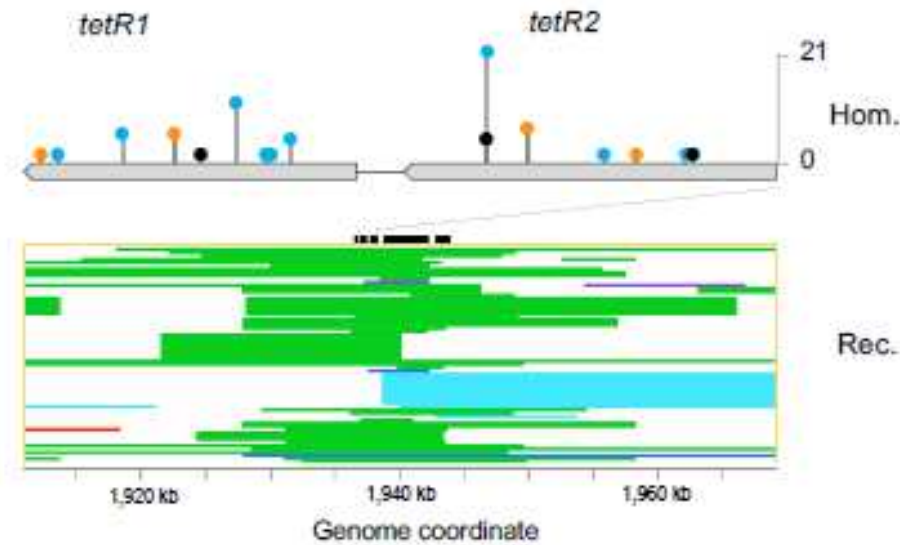

\section{Figure 5}

Genes under positive selections in the MKMC. A. Circular plot of genes under potential positive selection. Innermost inward bars, number of recombination per gene; innermost outward bars, number of mutations per gene (red bars, $n \geq 4$ ); outer red lines, location of highly polymorphic genes; outer blue line, location of genes with convergent mutations. B. Schematic diagrams depicting the distribution and frequency of non-synonymous mutations in zur and tetR1/2, and the recombinations around tetR1/2. Hom., homozygous mutation; Het., heterozygous mutation; Rec., recombination (strip colors represent donor subspecies corresponding to Figure 2). C. Schematic representation of mutations in LOS biosynthesis genes and corresponding morphology of the mutant strains. Genes were colored according to their functions. Green, genes involved in polyketide synthesis; orange, acyltransferase; cyan, glucosyltransferase.

\section{Supplementary Files}


This is a list of supplementary files associated with this preprint. Click to download.

- Supplementarylnfo.pdf

- Supplementarytables.pdf 